Historic, Archive Document

Do not assume content reflects current scientific knowledge, policies, or practices. 



\subsection{5}

Crawtord Gardens

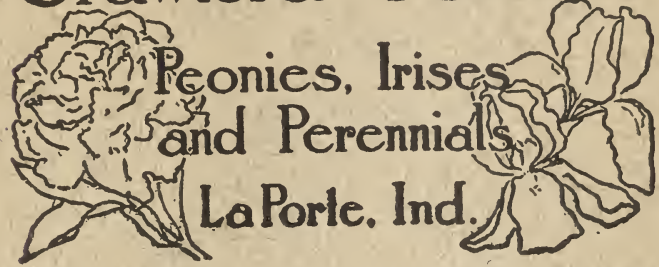

1927 


\section{Please Read Carefully}

We are including our price list of Peonles in this catalogue because our old customers have had our descriptive list. We will gladly send it to anyone who has not had it or who has lost it, if they will ask us for it.

The prices quoted are for strong, natural divisions of two, three or more eyes and no small plants will be offered, as they have been found unsatisfactory.

These prices include free packing but we do not pay the transportation charges. We will ship by express unless asked to send by parcel post. If customers prefer the package sent by parcel post, please send ten cents additional for each root or division and we will pay the postage and insure the safe delivery of the package.

All roots are guaranteed to be true to name, from healthy stock which has bloomed and been checked carefully by us. Occasionally a mistake may occur in digging and labeling and if such a case should happen we will replace the plant or return the money paid.

Orders are filled as soon after the first of September as growing conditions permit, exactly in the order in which they are received and as the quantity of some of the choicer varieties is limited we advise placing your order early.

\section{TERMS}

Our terms are cash with the order, and we will promptly return the money for any varieties of which we have sold out. We will not substitute.

NOTE-Many varieties are described in our catalogue which are not listed for sale this year. Should any of these be de. sired ask about them. 
Crawtord Gardens

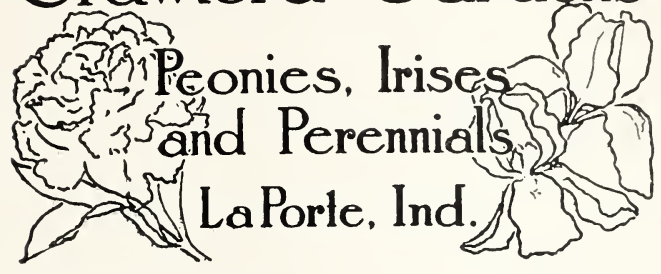

Mrs. Wm. Crawford, Prop.

2016 Indiana Ave.

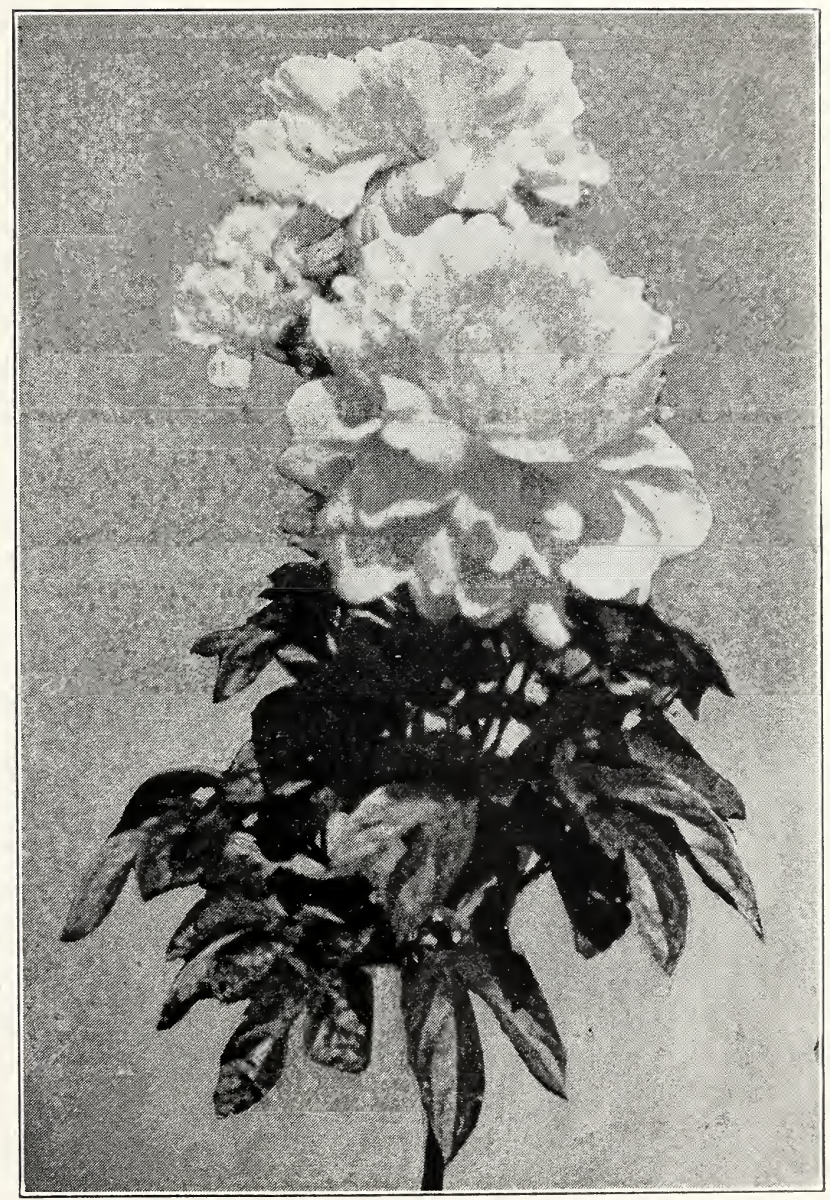

Therese 


\section{Peony Price List}

86 Addielanchia (Brand)

$\$ 10.0$ n

Adelaide E Hollis (Hollis) .................... 3.00

Adolph Rousseau (Dessert \& Mechin) ............... 1.50

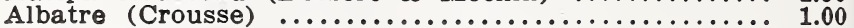

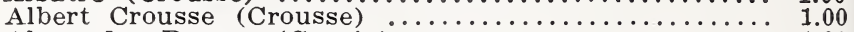

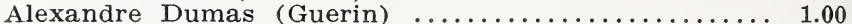

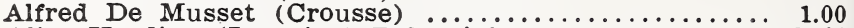

Alice Harding (Lemoine) Pale pink.............. 75.00

74 Alpheus Hyatt (Richardson) $\ldots \ldots \ldots \ldots \ldots \ldots \ldots \ldots \ldots \ldots . \ldots \ldots \ldots$

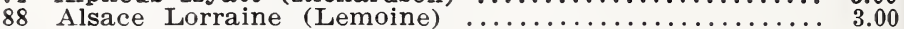

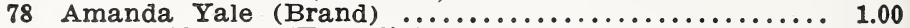

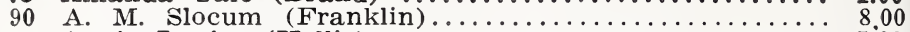

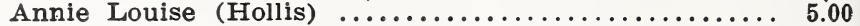

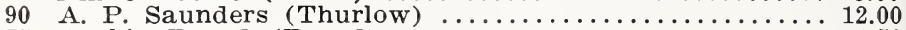

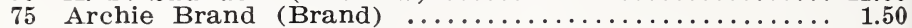

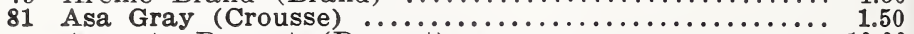

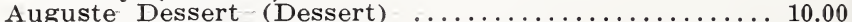

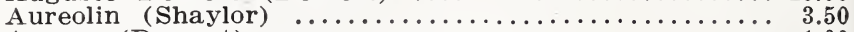

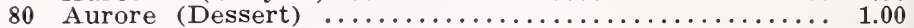

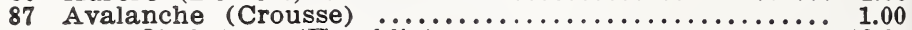

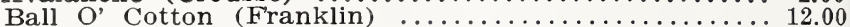

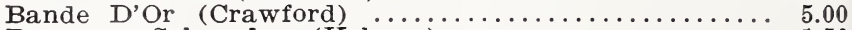

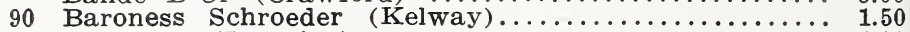

87 Bayadere (Lemoine) ....................... 4.00

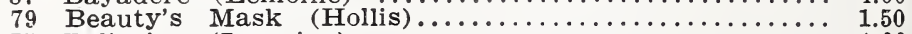

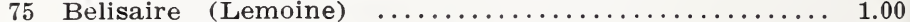

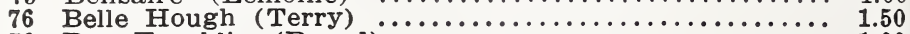

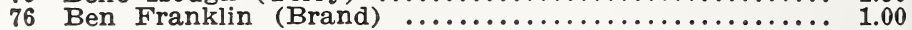

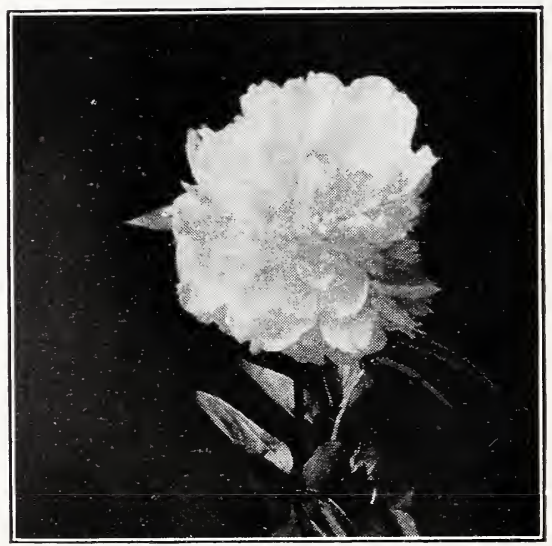

Cornelia Shaylor

Biebrich (Goos \& Koenemann) ............... 5.00

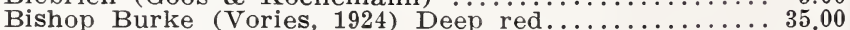

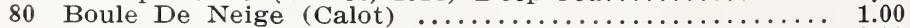

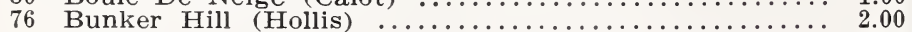

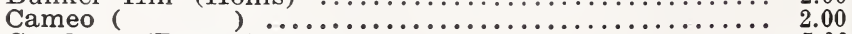

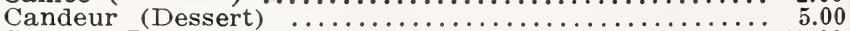

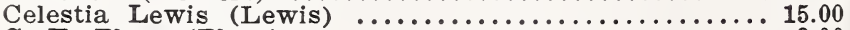

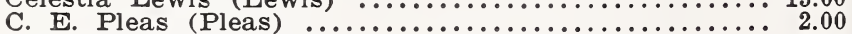

78 Charles McKellip (Brand) 2.50

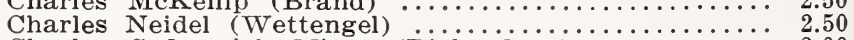

72 Charles Sedgewick Minot (Richardson)............... 2.00

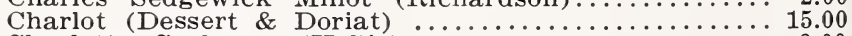

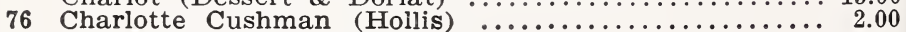

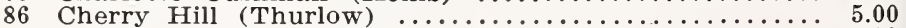

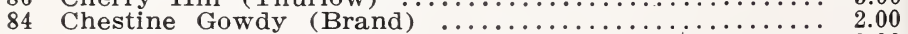

73 Christine Ritchie (Hollis) ................... 3.00

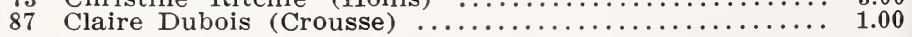


Clara Barton (Terry).

Clemenceau (Dessert)

Conqueror (Hollis)

A 1925 favorite . ......... 1.50

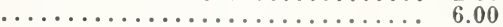

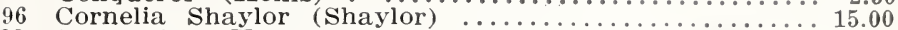

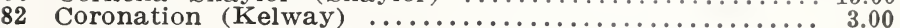

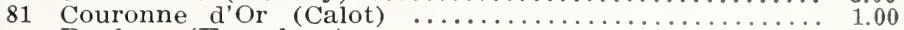

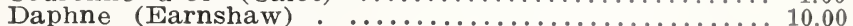

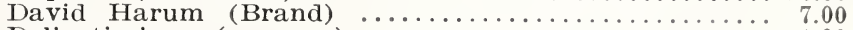

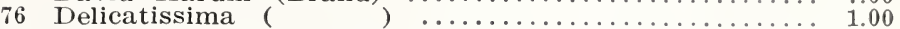

77 Dorchester (Richardson) ................... 1.00

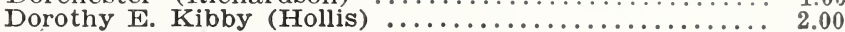

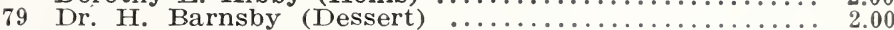

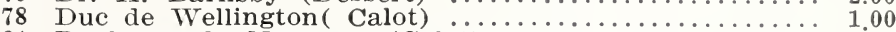

81 Duchesse de Nemours (Calot) 1.00

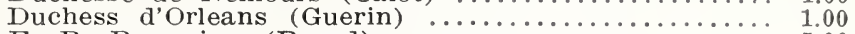

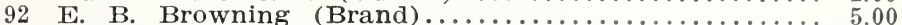

E. C. Shaw (Thurlow) Clear shell pink........... 20.00

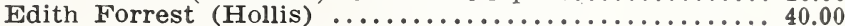

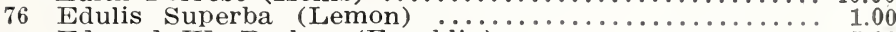

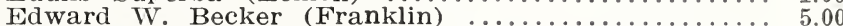

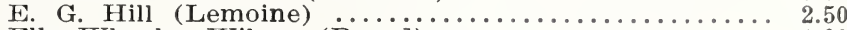

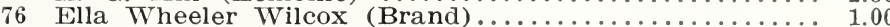

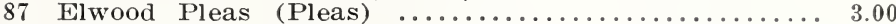

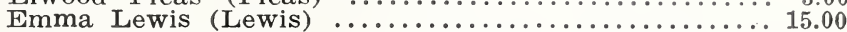

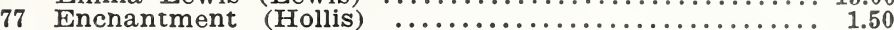

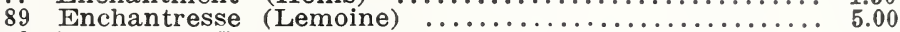

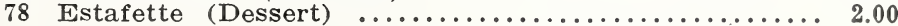

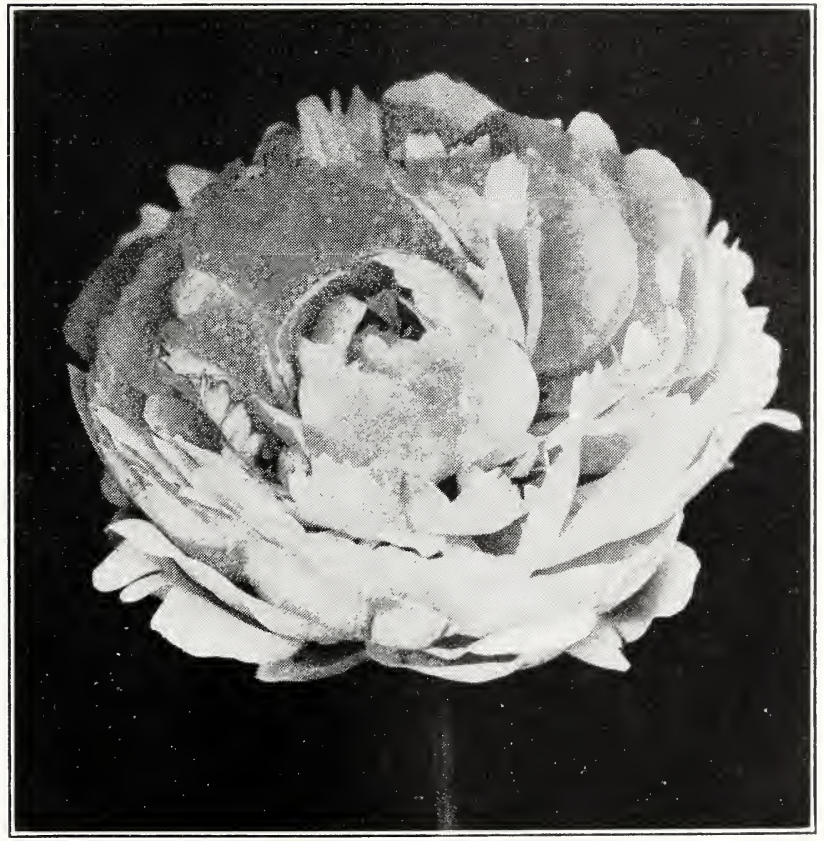

Georgiana Shaylor

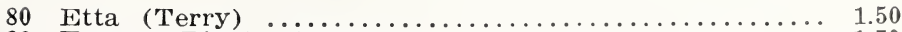

83 Eugene Bigot (Dessert)

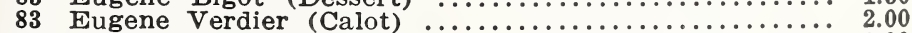

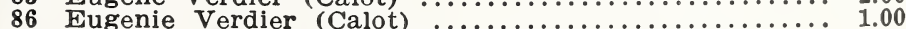

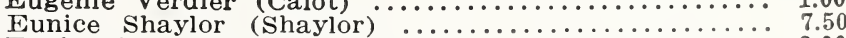

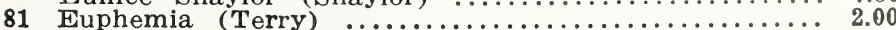

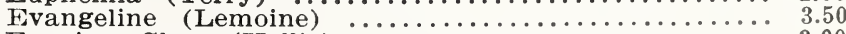

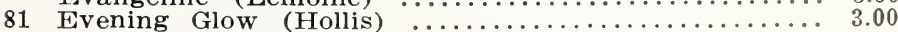




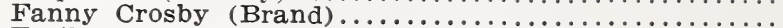

Faribault (Brand)

Felix Crousse (Crousse)

Ferdinand Stoliczka (Richardson)

Festiva (Donkelaer)

Festiva Maxima (Miellez)

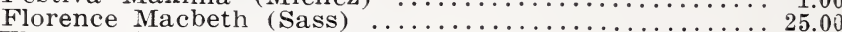

rence Nightingale (Brand)

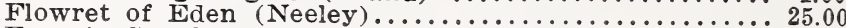

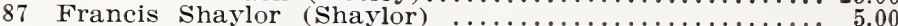

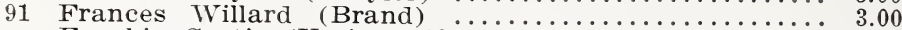

Frankie Curtis (Vories, 1924) White. tinted pink..... 35.00

Gen. Georgas (Van Leeuwin) Large, pure white........

George Hollis (Hollis)

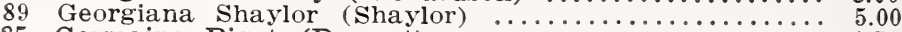

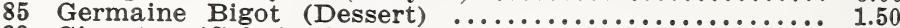

82 Gigantea (Calot) (Syn. Lamartine) $\ldots \ldots \ldots \ldots \ldots \ldots \ldots \ldots . . \ldots$

88 Ginette (Dessert)

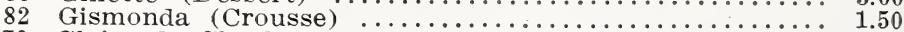

79 Gloire de Charles Gombault (Gombauit) .............. 1.00

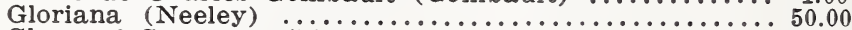

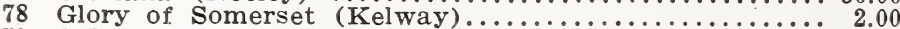

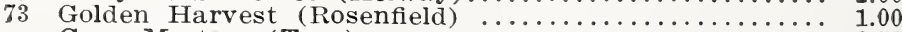

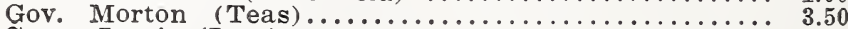

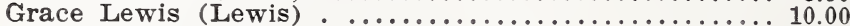

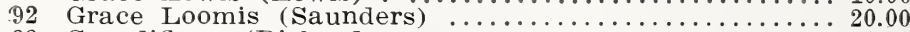

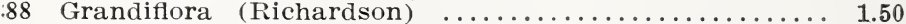

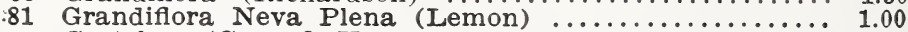

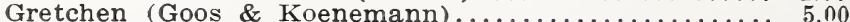

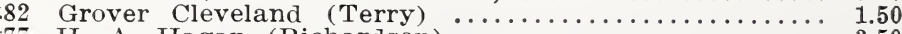

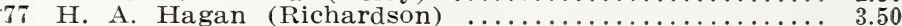

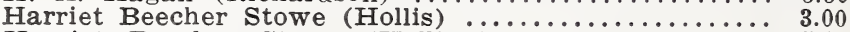

Harriet Beecher Stowe (Hollis-Anderson)............ 15.00

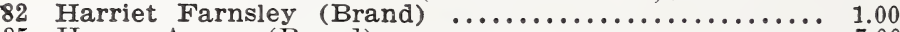

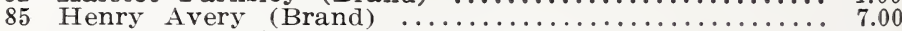

Henry M. Vories (Vories) Late, light pink........ 25.00

88 Henry Woodward (Richardson) (Grandiflora) ......... 1.50

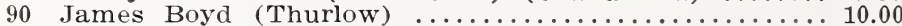

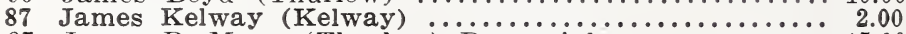

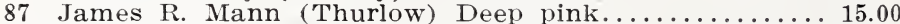

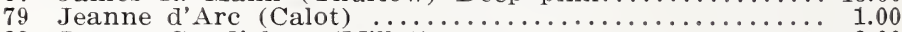

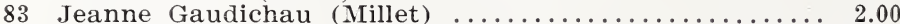

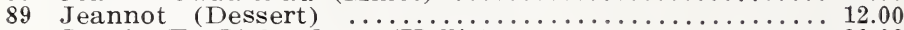

Jennie E. Richardson (Hollis)................ 20.00

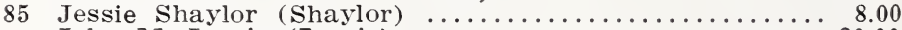

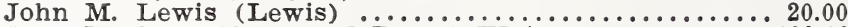

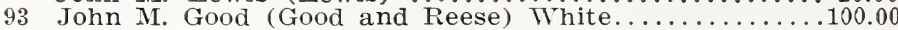

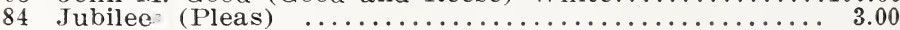

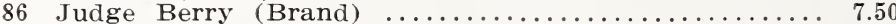

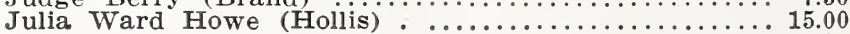

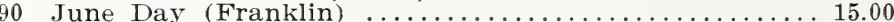

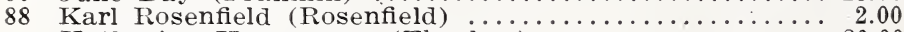

Katharine Havemeyer (Thurlow) ...............20.00

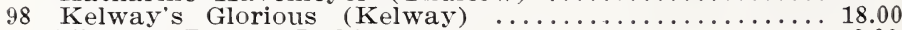

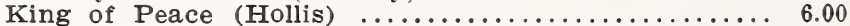

91 Lady Alexandra Duff (Kelway) . .............. 4.00

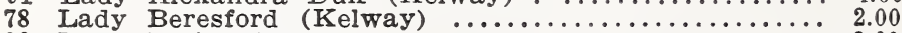

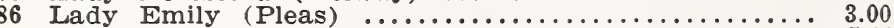

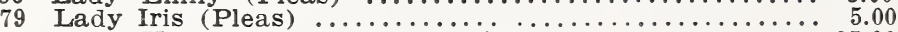

Lady Kate (Vories) Mauve pink................ 35.00

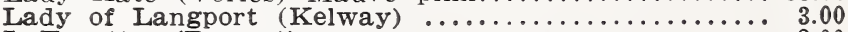

76 LaFayette (Dessert)

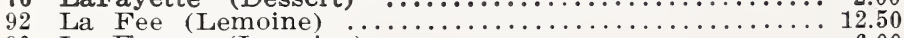

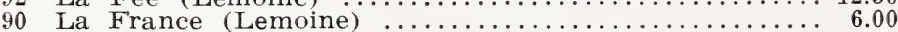

91 La Lorraine (Lemoine) .................... 8.00

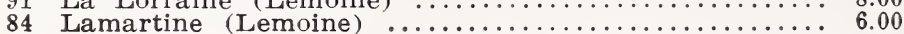

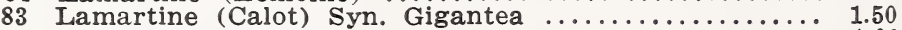

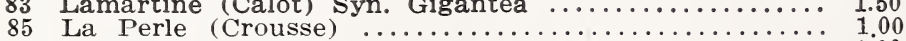

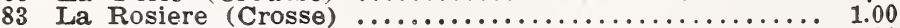

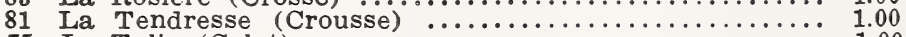

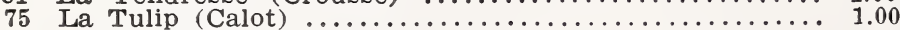

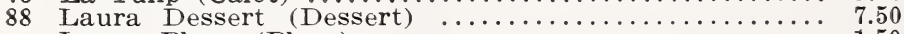

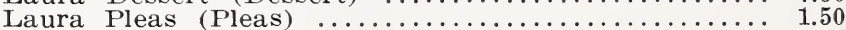

Laura Vories (Vories) White and bi ish pink......... 25.00

La Verne (Kelway) (Pink Baroness Schroeder) . ..... 5.00

99 Le Cygne (Lemoine) ...................... 10.00

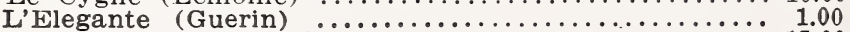

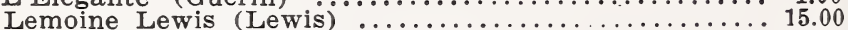

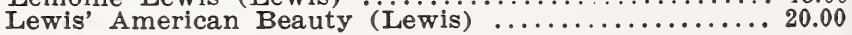


Lillian Gumm (Gumm)

81 Livingstone (Crousse)

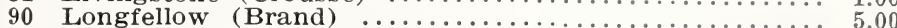

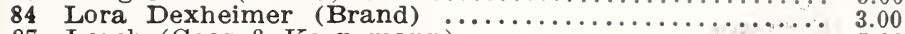

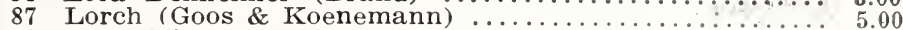

76 Lord Kitchener (Renault) .................. 2.00

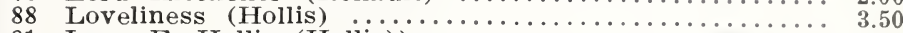

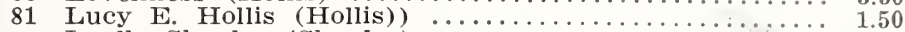

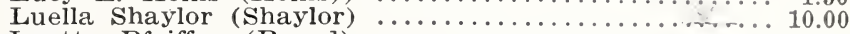

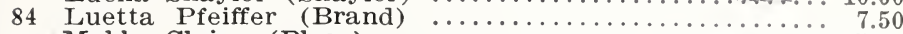

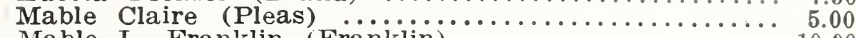

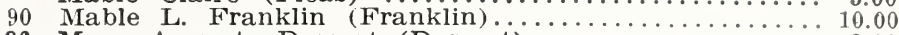

86 Mme. Auguste Dessert (Dessert) ........................... 2.00

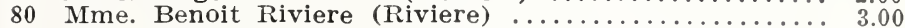

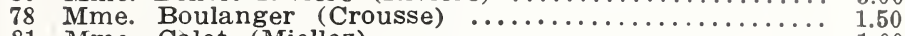

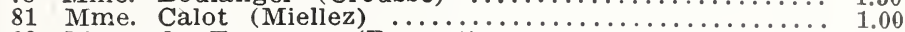

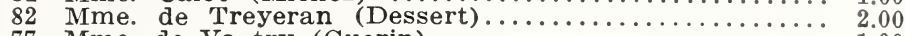

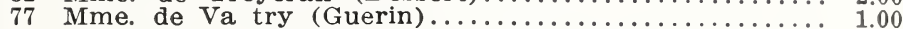

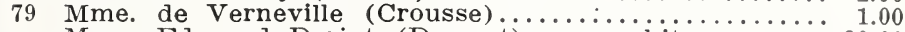

Mme. Edouard Doriat (Dessert) pure white........... 20.00

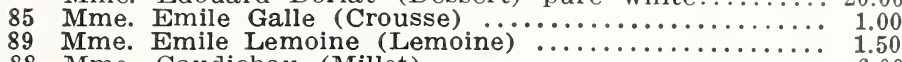

88 Mme. Gaudichau (Millet) ................... 6.00

83 Mme. Joanne Sallier (Paillet) $\ldots \ldots \ldots \ldots \ldots \ldots \ldots \ldots \ldots . \ldots \ldots$

94 Mme. Jules Dessert (Dessert).................. 5.00

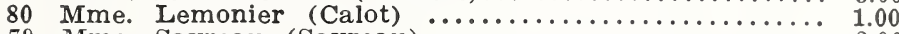

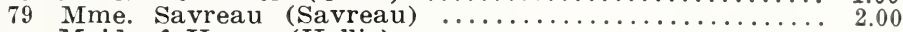

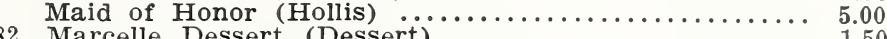

$\ldots \ldots \ldots \ldots \ldots+1.50$

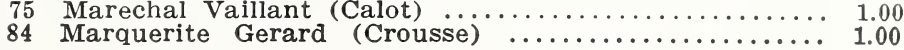

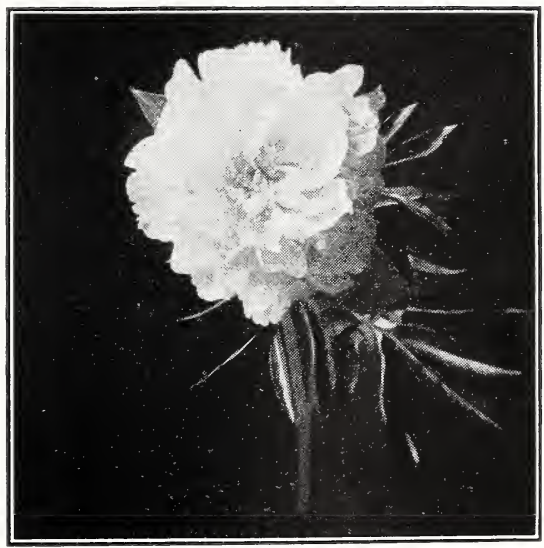

Miss Salway

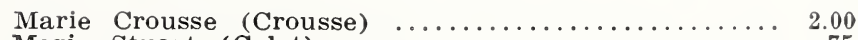

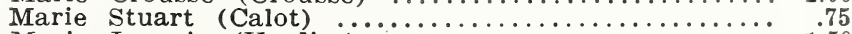

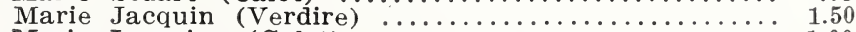

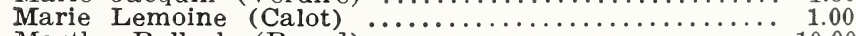

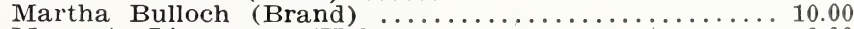

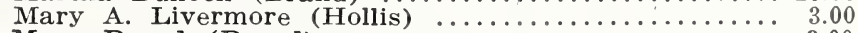

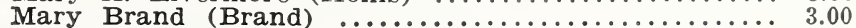

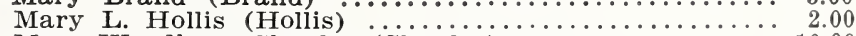

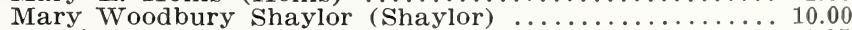

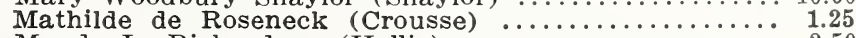

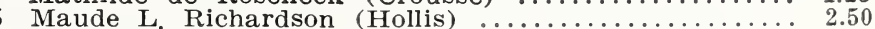

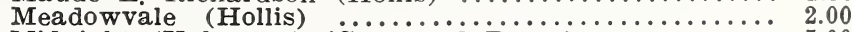

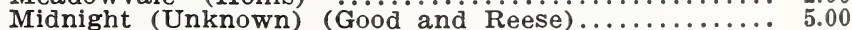

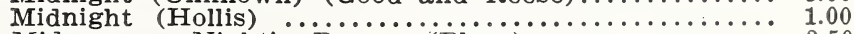

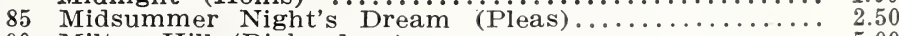

90 Milton Hill (Richardson) ................... 5.00

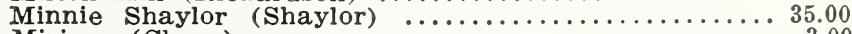

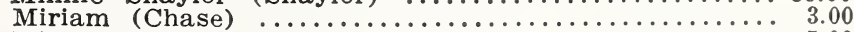

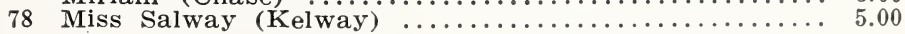


Mlle. Jeanne Riviere (Riviere) ................ 3.00

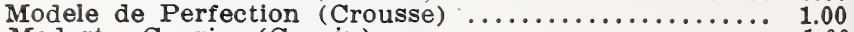

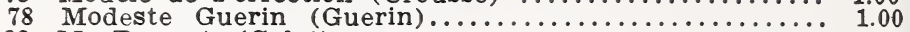

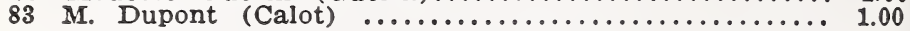

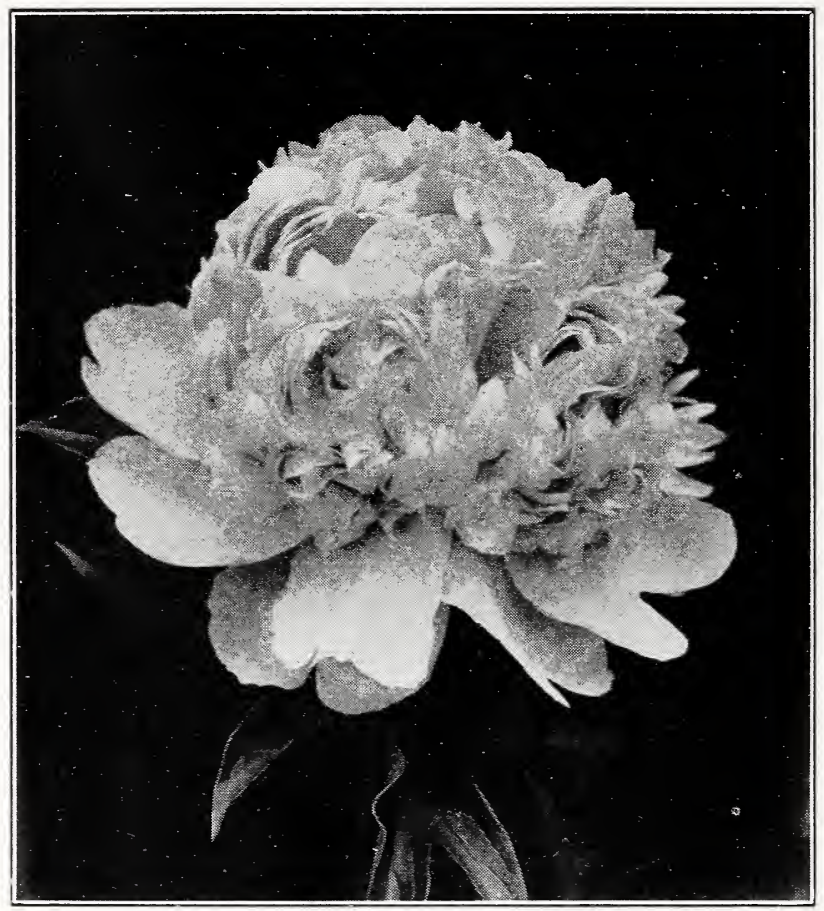

M. Jules Elie

M. Jules Elie (Crousse) ..................... 1.50

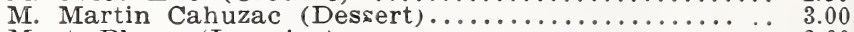

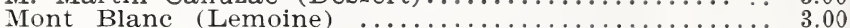

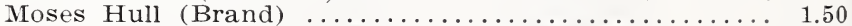

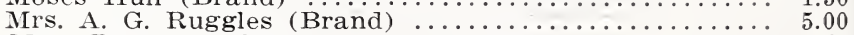

Mrs. Barrett (Pleas)........................ 6.00

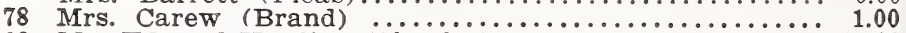

93 Mrs Edward Harding (Shaylor) .................. 15.00

Mrs. Jennie R. Gowdy (Brand) .................. 3.00

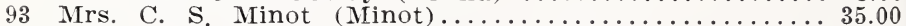

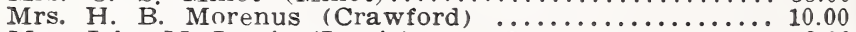

Mrs. John M. Lewis (Lewis) ................... 3.00

84 Mrs. John SmytheFogg (Hollis) ............... 3.00

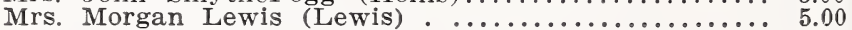

Mrs. M. P. Clough (Shaylor) ............... 4.00

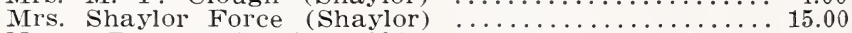

Nancy Dolman (Vories, 1924) very large pink........ 35.00

Nanette (Dessert \& Doriat) Pinkish white.......... 10.00

Nance O'Neal (Hollis) ........................ 5.00

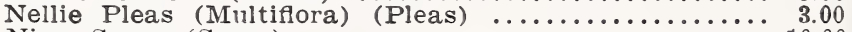

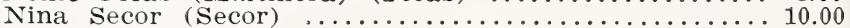

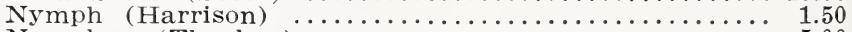

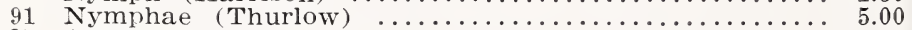

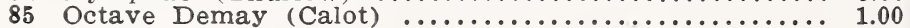

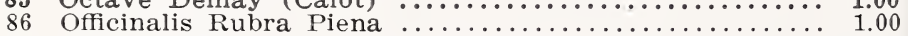

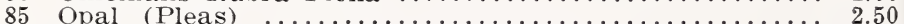

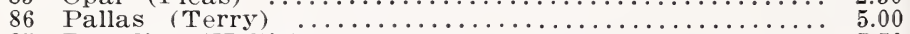

85 Paradise (Hollis) ....................... 7.50 


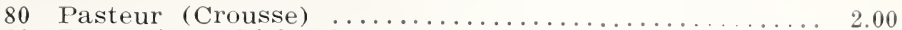

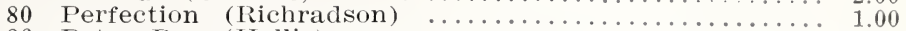

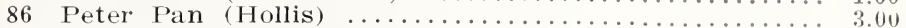

92 Phillippe Rivoire (Riviere) ..................... 12.50

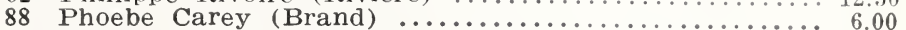

88 Phyllis Kelway (Kelway) .................. 10.00

82 Pierre Duchartre (Crousse) ....................... 2.00 Pink Baroness Schroeder-See La Verne.

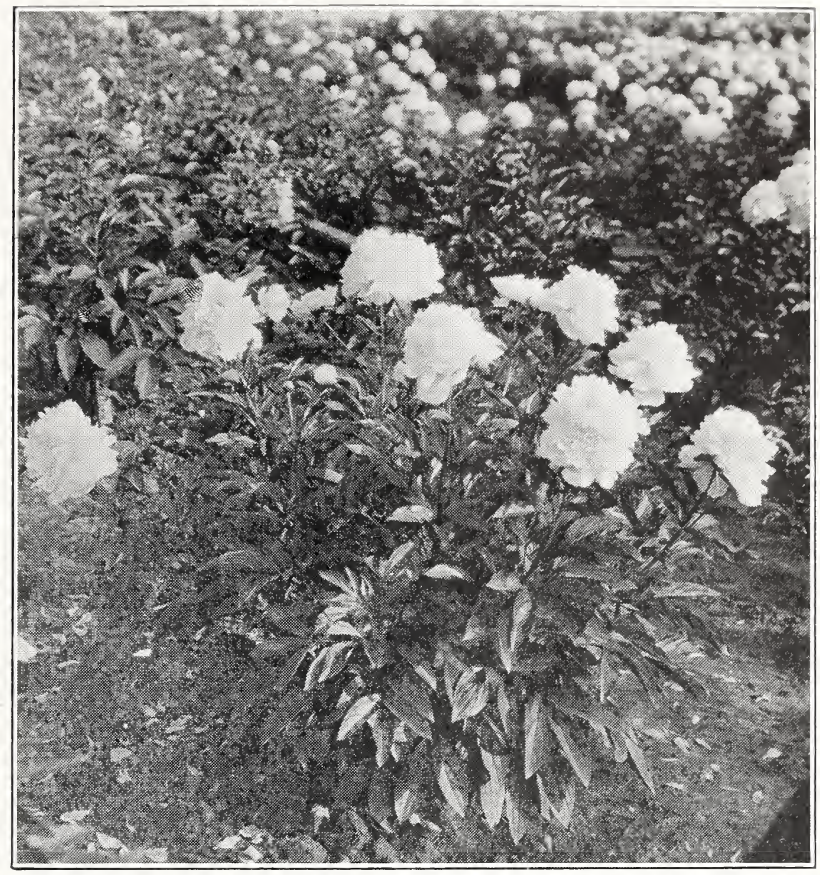

Mrs. C. S. Minot

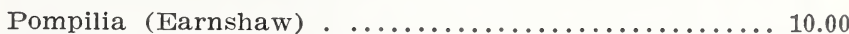

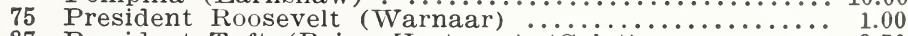

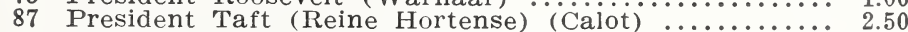

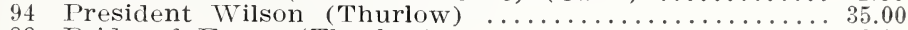

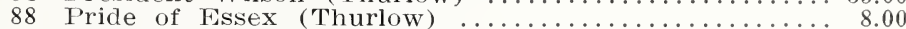

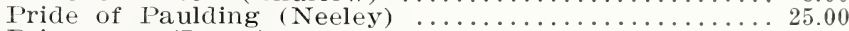

86 Primevere (Lemoine) ..................... 3.00

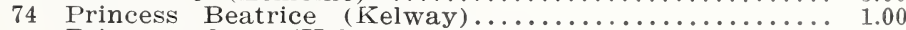

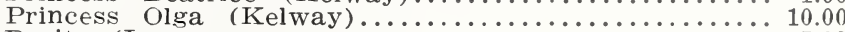

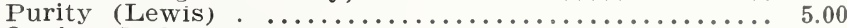

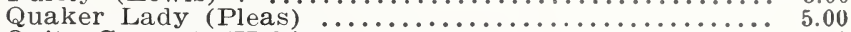

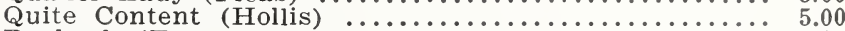

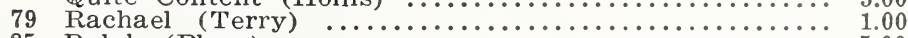

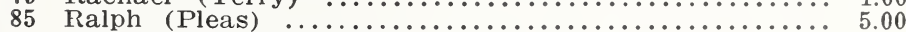

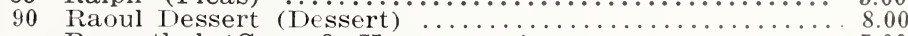

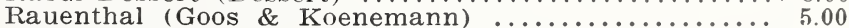

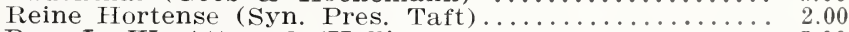

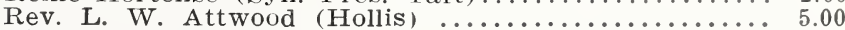

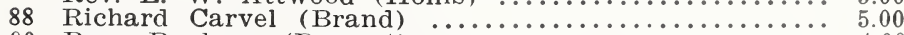

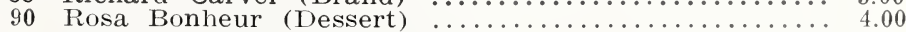

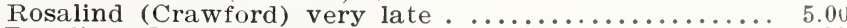

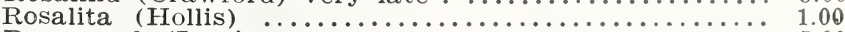

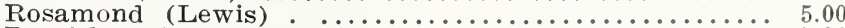

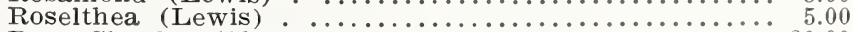

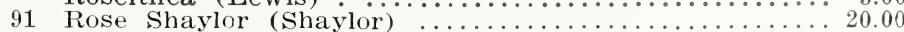

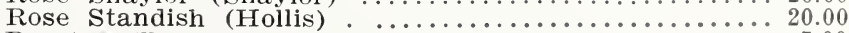

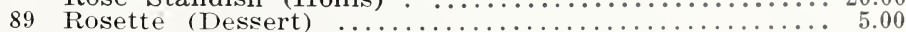

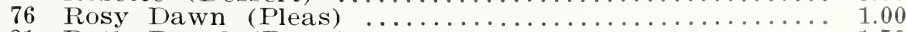

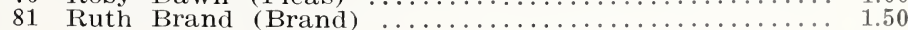

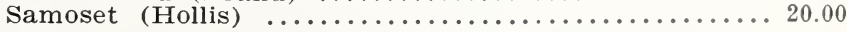


82 Sarah (Pleas)

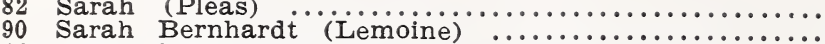

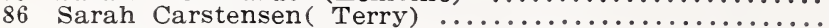

91 Sarah K. Thurlow (Thurlow)

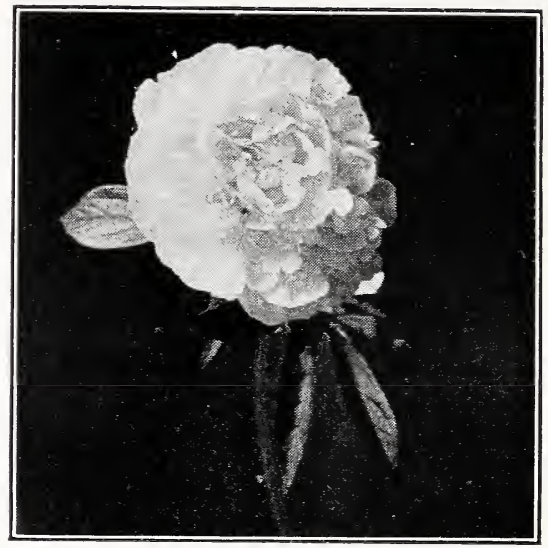

Rose Shaylor

Sarah M. Wettengel (Wettengel)................ 5.00

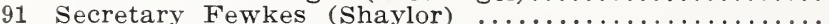

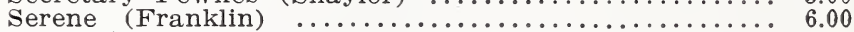

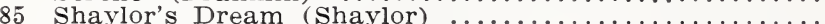

79 Simone Chevalier (Dessert) $\ldots \ldots \ldots \ldots \ldots \ldots \ldots \ldots \ldots \ldots$

97 Solange (Lemoine) $\ldots \ldots \ldots \ldots \ldots \ldots \ldots \ldots$

Sops of Wine (Secor) $\ldots \ldots \ldots \ldots \ldots \ldots \ldots \ldots \ldots$

1.00

4.00

5.00

3.00

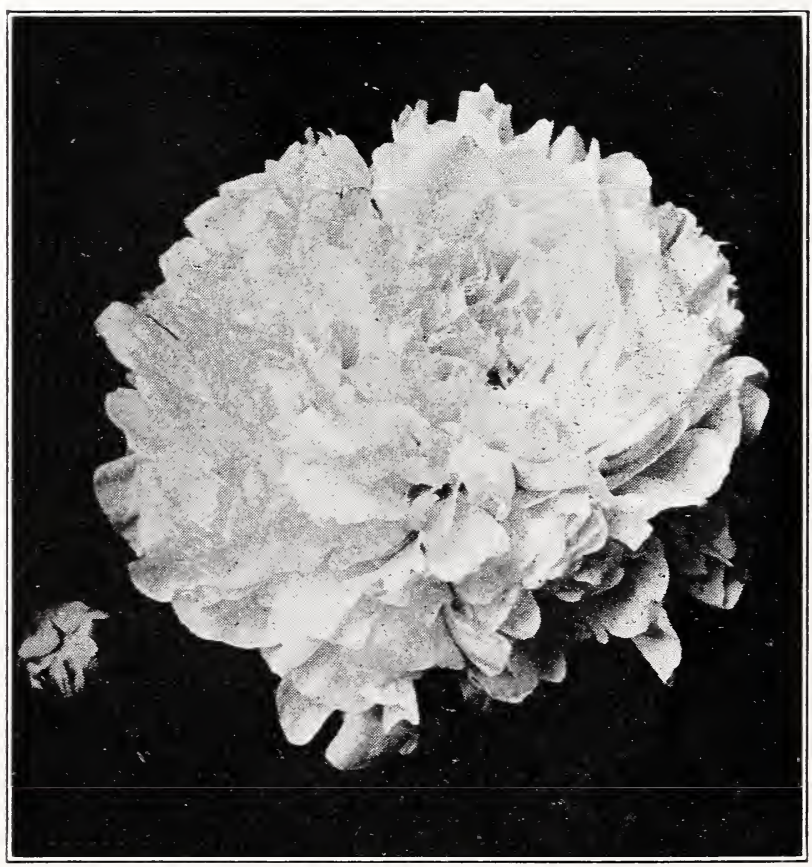

The Eagle 


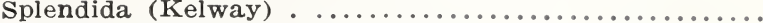

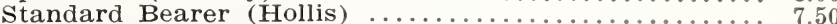

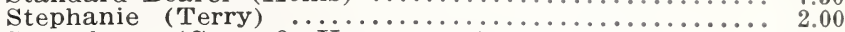

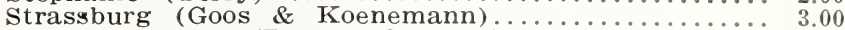

Suzanne Dessert (Dessert \& Mechin) ............. 1.00

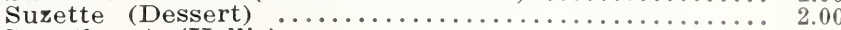

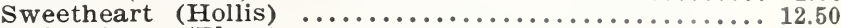

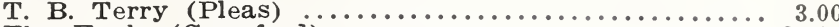

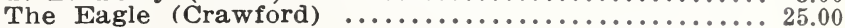

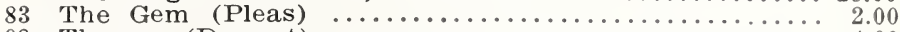

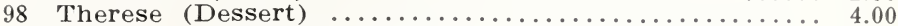

91 Thomas C. Thurlow (Thurlow) Delicate salmon-flesh.. 25.00

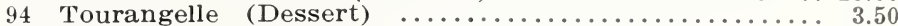

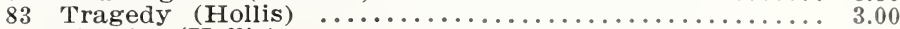

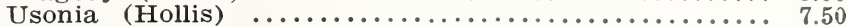

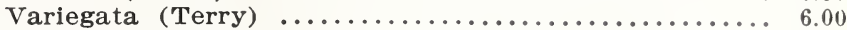

Venus (Kelway)

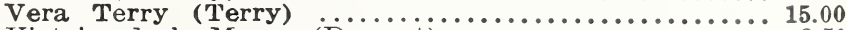

Victoire de la Marne (̇̈.

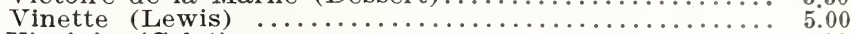

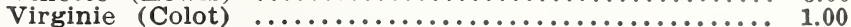

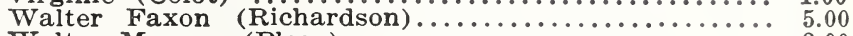

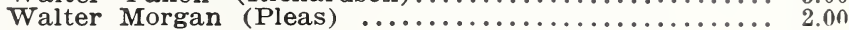

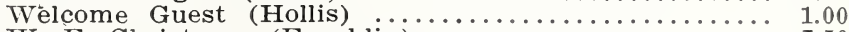

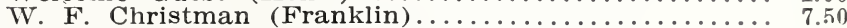

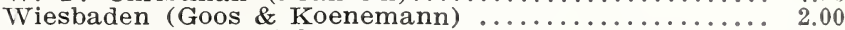

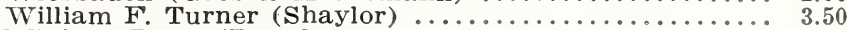

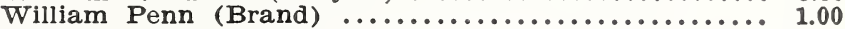

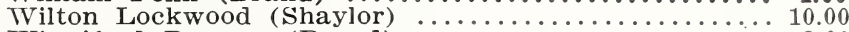

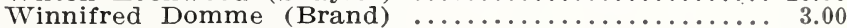

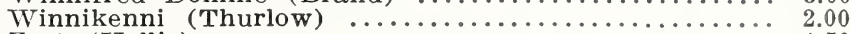

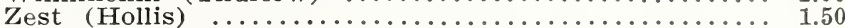

Tree Peony (Paeonia Moutan) Pink.......... $\$ 3.00$ to 5.00

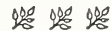

\section{JAPANESE}

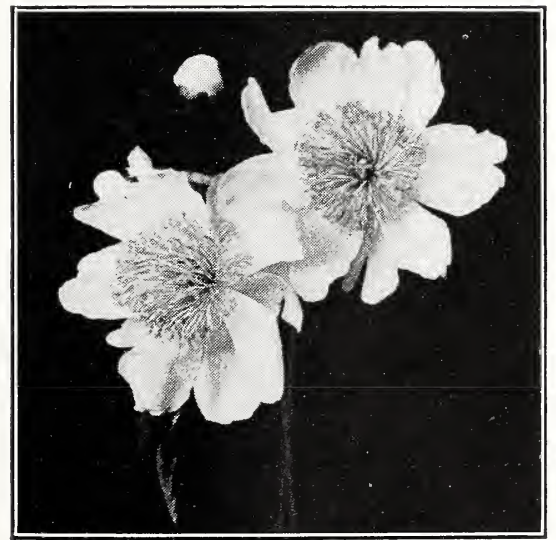

Minot No. 4 A

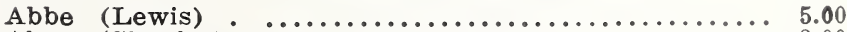

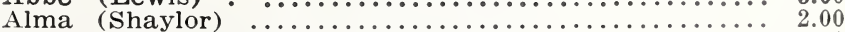

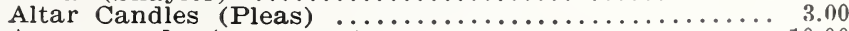

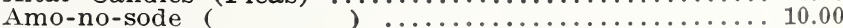

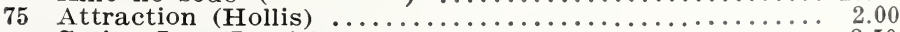

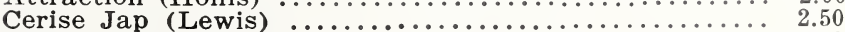

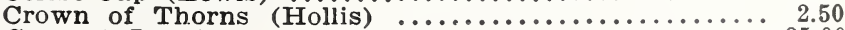

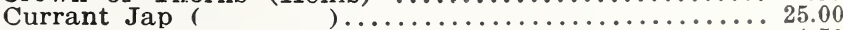
Dr. Edward Pleas (Pleas) Syn. Ostrich Plume.......... 1.50

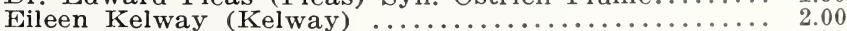

77 Esther (Terry)

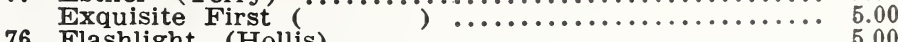

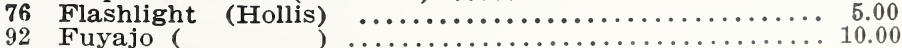




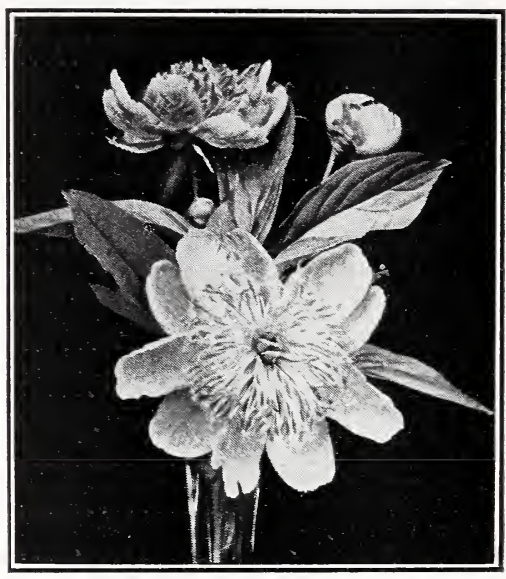

Goldmine

Goldmine (Hollis)

3.00

Gypsy (Hollis) .

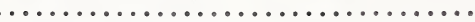

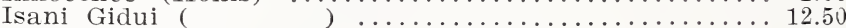

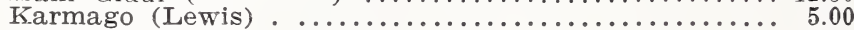

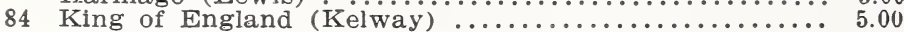

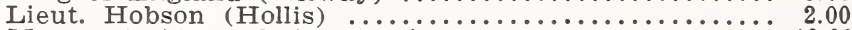

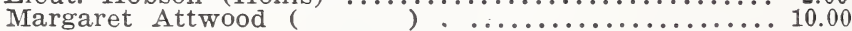

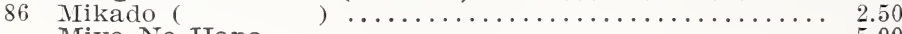

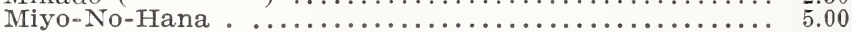

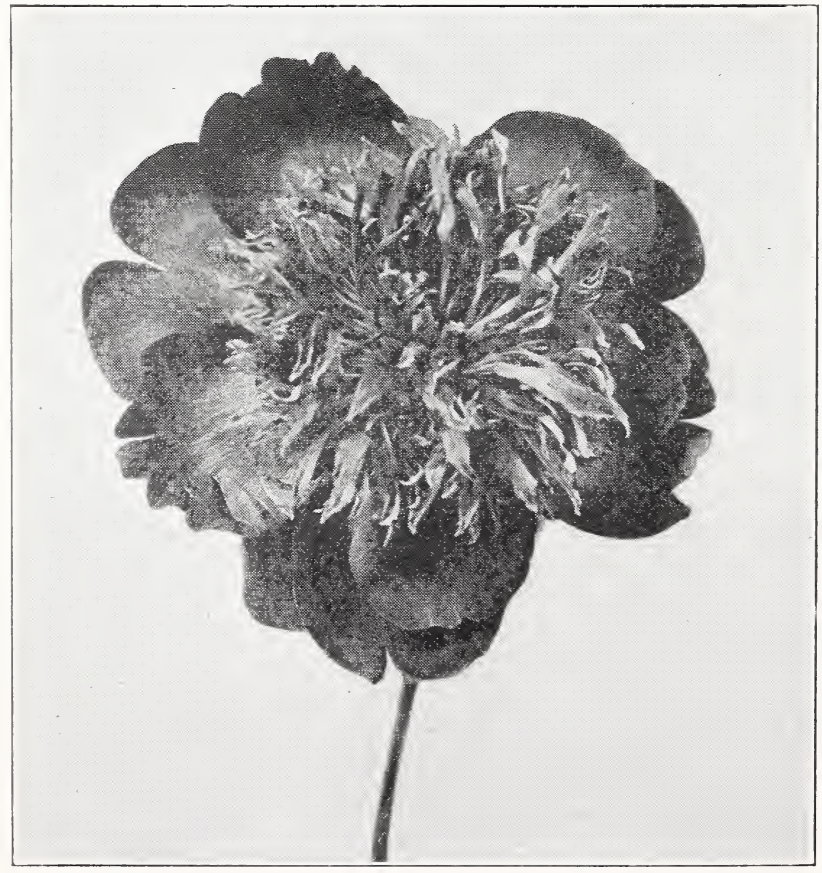

Prince Ito 


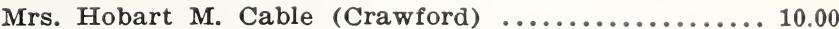

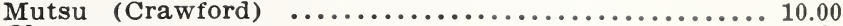

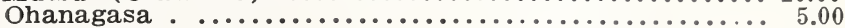

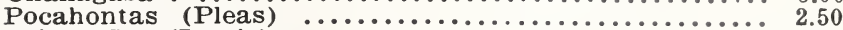

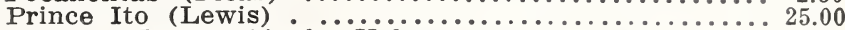

Princess Duleep Singh (Kelway) ................ 2.50

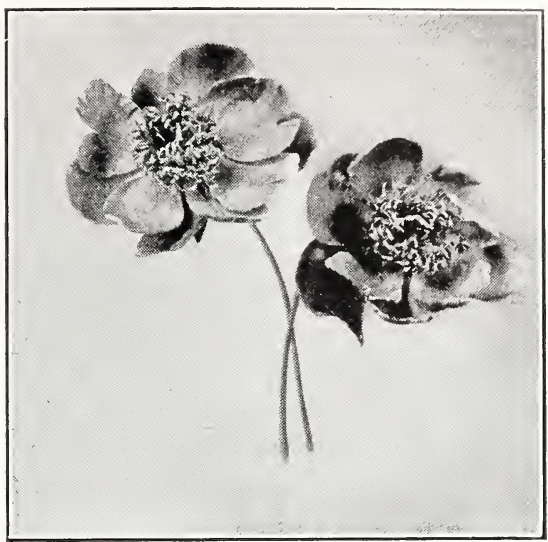

Queen Alexandria

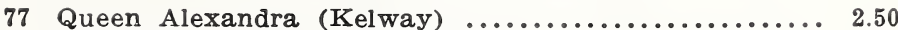

Sir Madho Singh (Kelway) $\ldots \ldots \ldots \ldots \ldots \ldots \ldots \ldots \ldots \ldots \ldots . . \ldots \ldots . \ldots \ldots . \ldots \ldots$

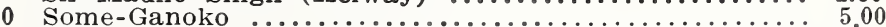

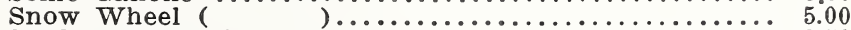

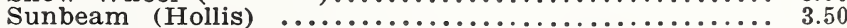

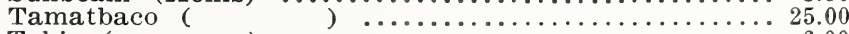

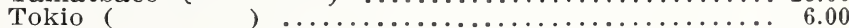

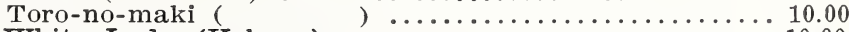

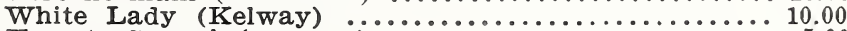

Yamato-Sangai ( $)$..................... 5.00

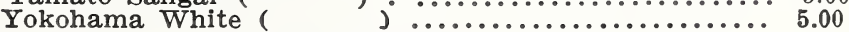

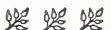

\section{SINGLE}

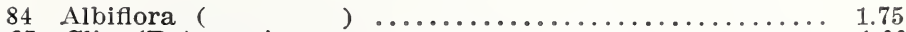

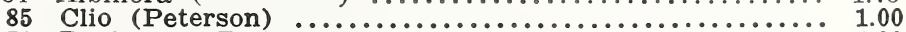

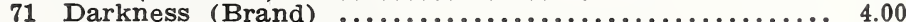

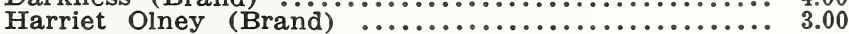

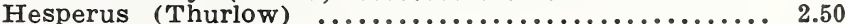

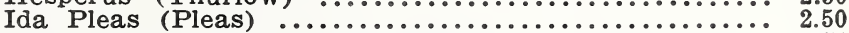

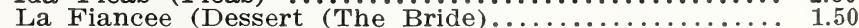

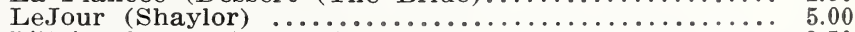

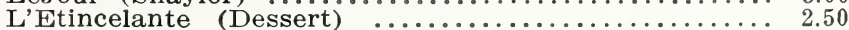

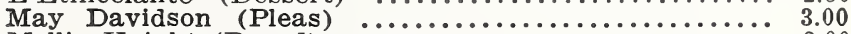

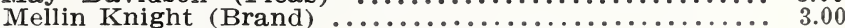

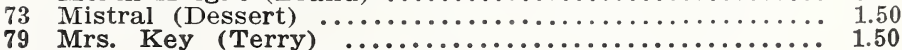

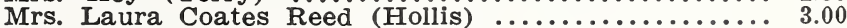

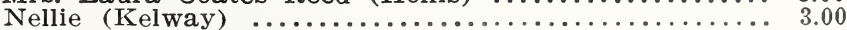

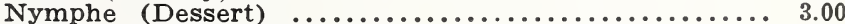

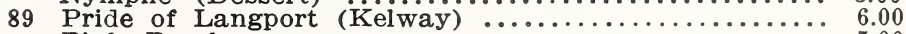

Pink Pearl

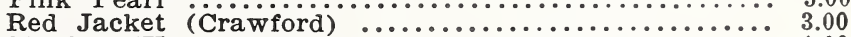

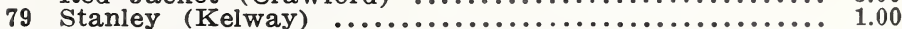

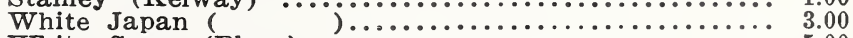

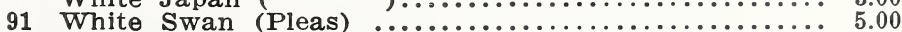

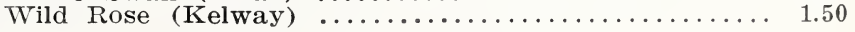

Collections of 10 peonies, our selection, will be furnished for $\$ 5.00$ and will make a good hedge or a row from which to cut blossoms for the house. 


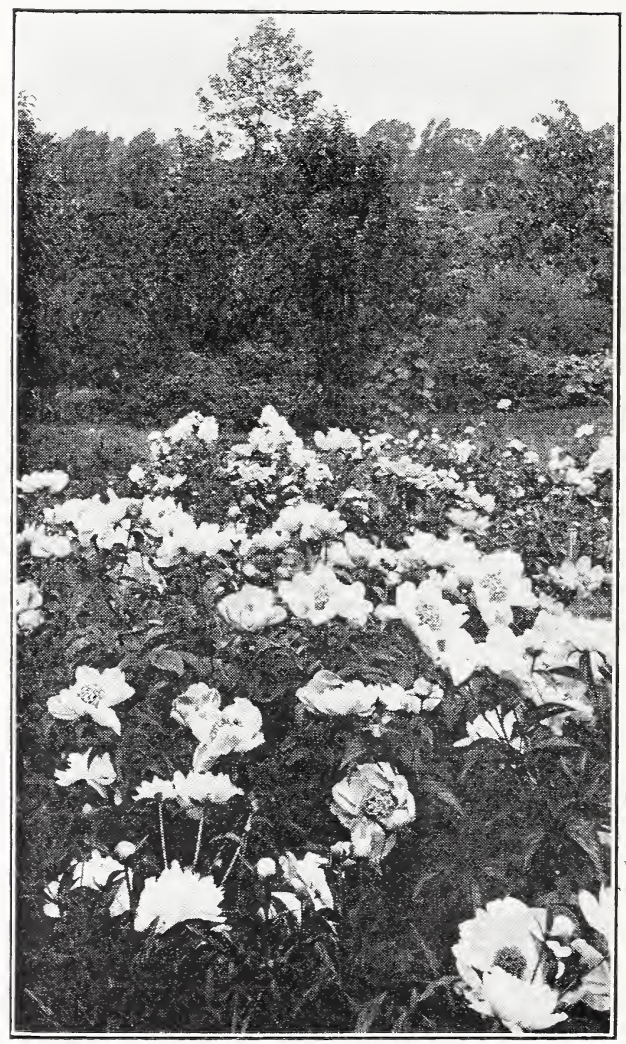

A Border of Single Peonies 


\section{IRISES}

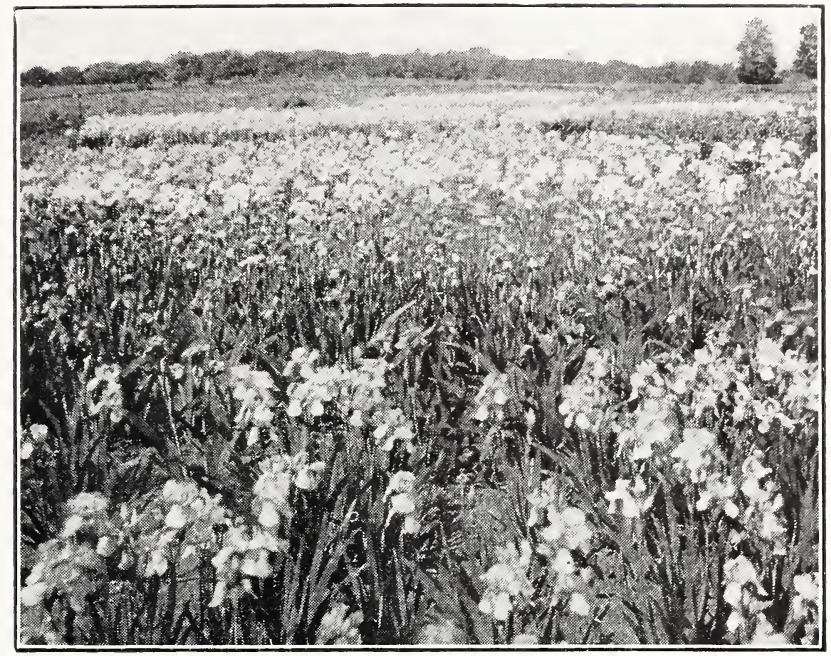

There is probably no garden flower that has the great range of color of the Iris; blue, yellow, white, red and intermediate shades make it possible to obtain almost any desired effect, and this fact, together with the ease with which the Iris is grown, is undoubtedly responsible for the increasing popularity of this flower. The name Iris is derived from the Greek, meaning Rainbow, and certainly every color of the rainbow has been caught and held in the petals of this flower.

They can be used anywhere. The dwarf varieties are quite adaptable to the rock garden; the taller hybrids can be massed in any garden, including the formal one, and the Japanese and Siberian varieties are wonderfully effective near or around the water. The foliage lasts well and the glaucous green of the leaves makes a restful patch of color when the plants are not in bloom. With a bit of care in selecting varieties, continuous bloom for at least thirty days can be arranged. If you have never had the thrill of going out into a bright spring morning to see for the first time an Iris which opened during the night or at dawn, you have missed the spirit of Spring.

How easily Irises are grown, compared with almost any other flower. Sunlight and an ordinary well drained garden soil, not overly rich, are all that they require. Irises do not like manure and if fertilization seems needed a small amount of bone beal, mixed with limestone, can be stirred in around the roots. They are best transplanted soon after the plants are through flowering, in July and August, when they soon establish themselves well enough to go through the winter without being heaved by the frost. If planted in the fall a light mulch will protect 
them from the lifting action of the frost, but it is well to set them a little deeper than for summer planting. The soil should be prepared as carefully as for any seed bed and if possible allowed to settle. Then dig a wide, shallow trench and spread the long roots out in a natural position but only just covering the bulb. Pack the earth well with the hand, and water. The water will continue the packing process and also leave the top of the bulb exposed to the sun as it should be. Plants should only be reset after they have become so crowded that they no longer bloom well. Cultivation will improve the looks of the garden and increase the vigor of the plants.

Two diseases are noticed among Irises: the borer and the rot. The borer is a small worm, hatched about blooming time, from an egg laid on the leaves by a night flying moth. The worm, increasing in size, gradually eats its way down the stalk between the leaves, which are quickly discolored. During this period the worm can be easily detected and destroyed by pulling the leaves apart. If the worm reaches the bulb, it eats out the inside while developing into a creamy-white grub about an inch and one quarter long, causing rot and sometimes the loss of many blossom buds. When a planting becomes infected in this way the bulbs must be lifted, thoroughly cleaned and reset. Watch the foliage and destroy the worms while it is still easy. We have three colonies of Purple Martins established in houses at intervals over the field. These birds will feed upon the egg laying moth as it comes out in the early evening. To see and hear them while taking their food on the wing is an inspiration and to be free from the borer in our Iris gardens is a great satisfaction.

The rot seems to be caused by wet, soggy soil, due to poor drainage or an unusually wet season. If Irises are set far enough apart so that the sunlight can reach the soil and care is taken to provide good drainage there will be little chance for rot to develop. Acid soil may be a contributing cause, so an application of crushed limestone should always be given a sour soil.

In the following descriptive list of Irises we are offering most of the tried and proven varieties which appeal to the average garden owner. Some of the new and outstanding varieties are still so high in price that they can be considered only by the collecter; however, we are offering many of them at prices which are within the reach of the average flower lover and all of them have been tested in our rather severe climate.

The numbers which precede the names are the ratings of the varieties. These ratings are the results of votes taken by the members of the American Iris Society on the basis of a theoretically perfect Iris which would rate 100. A flower which rated 70 would be a good Iris and additional points indicate its approach toward perfection. The ratings can well be used as a guide in selecting varieties.

WE DO NOT SELL IRISES IN THE SPRING, as we have found that the growth is seriously retarded. We will ship in July and August when the plants are in the best condition for transplanting. Our prices are all based 
on single natural divisions, good strong rhizomes that ought to bloom the following season. Some varieties have much smaller rhizomes than others and this must be considered, but we always serd out the strongest possible plant according to the variety.

\section{CRAWFORD SEEDLINGS}

We are constantly growing and selecting new seedlings. Some we are holding until our stock is large enough to permit us to list them, but we will be glad to show them to visitors. We can now offer ANNETTE, which is distinct enough to be of value in any garden, and more will follow.

Mixed seedlings at $\$ 5.00$ per 100 .

\section{路路路 \\ TERMS OF SALE \\ Read carefully before ordering}

GUARANTEE:

We believe our stock to be absolutely true to name and do everything possible to avoid mistakes. Should any error occur we will gladly make it good if notified promptly after first blooming.

SHIPMENT:

Orders for Iris will be filled during JULY and AUGUST and in rotation as they are received unless a certain date is specified.

STOCK:

We ship only good strong rhizomes, the natural division of the Iris and one which ought to give bloom the first season.

TERMS :

Cash with the order. No order accepted for less than $\$ 1.00$.

CARRIAGE:

We do not pay transportation charges. Please tell use whether to ship by parcel post or express. If by parcel post, please include $10 \mathrm{c}$ for the first and $5 \mathrm{c}$ for each additional plant. We will then prepay the postage and, if there is a balance, return it to you.

PRICES :

Are net and not subject to discount.

ORDER EARLY:

As the quantity of many varieties is limited and you will be disappointed if we are sold out on them.

\section{路路路}

\section{THE AMERICAN IRIS SOCIETY}

A membership in the A. I. S. is a good investment and will give you much pleasure as well as valuable information and advice. The annual dues are but $\$ 3.00$ and checks made payable to the American Iris Society we will be glad to forward to the Secretary. 


\section{Irises}

(The figures preceding the name are the ratings of the American Iris Society on a score of 100 as perfection. Those without number have not as yet been rated).

S. refers to the standards which are the three upper petals.

F. refers to the falls or the three lower petals.

Self refers to a flower with both standards and falls of the same color.

83 AFTERGLOW-Sturtevant, 1917) A delicate grayish lavender self with rich yellow shades throughout the center. Strong flower. 40 inches.....75c each

AKSARBEN-(Sass, 1923) Fawn and brown markings on a yellowish ground. Very distinct and fine for a low border. 24 inches.........\$4.00 each

73 ALBICANS-Snow white self, very early and fragrant. 18 inches ...........25c each; $\$ 2.00$ per doz.

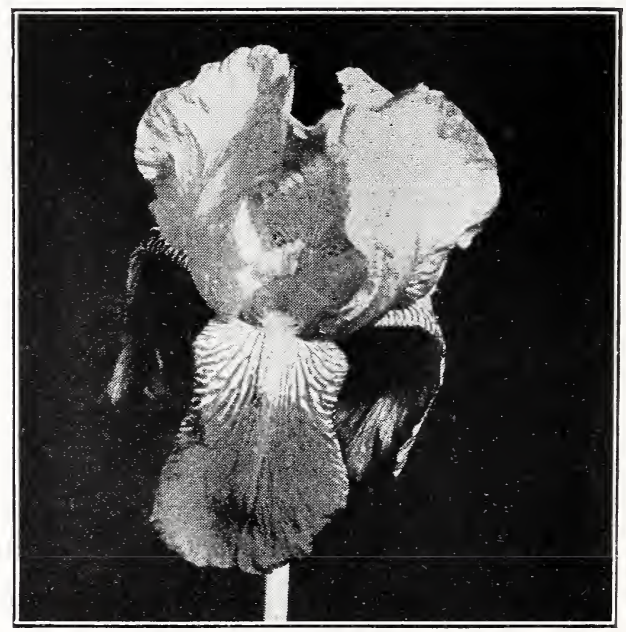

Alcazar

89 ALCAZAR-(Vilmorin, 1910) S. light bluish violet F. deep purple with bronze veined throat. A large, striking flower on a well branched, sturdy stem. 42 inches.............35c each; $\$ 3.00$ per doz.

94 AMBASSADOR-(Vilmorin, 1920) S. smoky reddish violet; $F$. dark, velvety reddish violet, held almost horizontal. A magnificent flower with tall stiff stems. Late. $36-40$ inches.........\$1.00 each

78 AMAS-(Species) S. rich blue; F. violet. A handsome flower from Asia Minor. 28 inches......

$35 \mathrm{c}$ each; $\$ 3.00$ per doz.

ANGELO-(Hort, 1920) S. pale lavender blue; F. deeper in tone, very long and broad. Very large flowers on strong, straight stems. 30-36 inches. 
86 ANN PAGE-(Hort, 1919) Pale lavender blue self, the long falls have brown pencilings at the haft. A large flower of fine shape. 36 inches....\$5.00 each

85 ANNA FARR-(Farr, 1913) S. white bordered with pale blue; F. white with blue base, held horizontal. Delicate in tone. 36 inches.....75c each

83 ANNE LESLIE-(Sturtevant, 1917) S. white with slight flush of rose; $F$. rich carmine, with orange beard. Unusual color tones. 30 inches....75c each

ANNETTE-(Crawford, 1925) White ground; standards heavily overshot with deep blue-purple markings. Falls edged same color and with a distinct line down the center. Medium size. 30 inches...............\$5.00 each

APHRODITE-(Dykes, 1922) The very best of the "pink" irises. The whole flower is a clear, translucent lilac pink, with no veinings. White haft, with a pale lemon beard. Vigorous and free bloom-

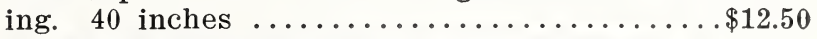

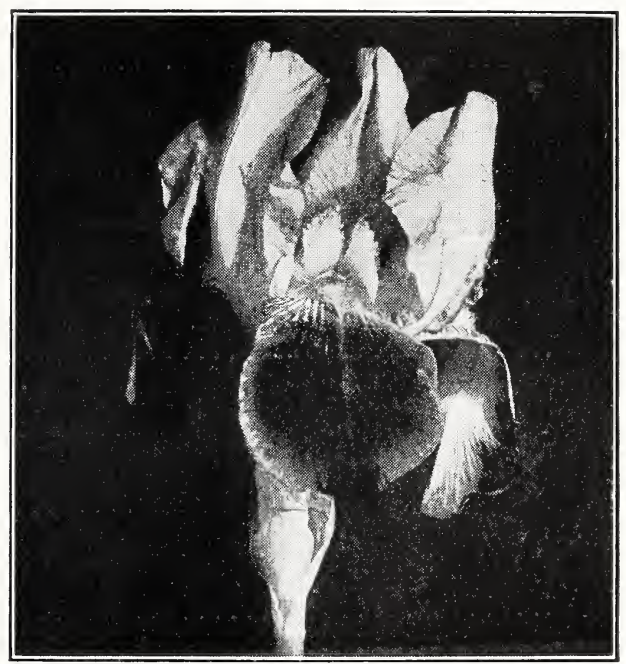

Ambassadeur

83 ARCHEVEQUE-(Vilmorin, 1911) S. deep velvety violet; $F$. dark velvety purple, immense flowers. $20-24$ inches ............35c each; $\$ 3.00$ per doz.

ARGENTINA-(Mohr, 1924) Pure white, with gold veinings on the haft and prominent yellow beard. There is nothing finer among the whites. About 30 inches ................... \$7.50

84 ARSACE-(Millet, 1913) Lavender pink self. Large flowers on 30 inch stems....50c each; $\$ 4.50$ per doz.

92 ASIA-(Yeld, 1920) The superb iris. S. pale lavender, shaded golden yellow at the base; F. light violet purple, with slightly lighter margins. Large flowers and tall stems; everything that a superlative iris should have. 48 inches .............\$4.00 
ATLAS-T. B. (Millet) S. lobelia blue, slightly ruffled F. blue, lined lilac............... \$1.00

74 AUREA-T. B. (Jacques, 1830) S. and F. clear golden yellow. A great favorite with all who see it in our garden. 30 inches...........35c; $\$ 3.50$ per doz.

AUSTIN-T. B. (Denis, 1923) S. and F. bright wine red with a yellow tinge. An effective Squalens variety ........................... $\$ 1.00$

AUTUMN KING-(Sass) Lavender violet to hyacinth violet on falls. Never fails to produce a second crop of blooms in the fall. Strong grower. 34

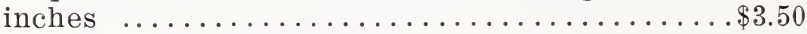

AYMARD-T. B. (Denis, 1922) S. palest shade of Bishop's violet, F. paler ground with distinct purple veinings. 30 inches ...............\$1.00

82 AZURE-T. B. (Bliss, 1918) S. lavender blue; F. rich violet blue, yellow beard. Free bloomer. 36

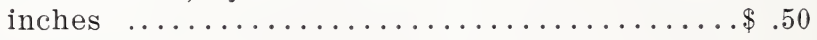

BALARUC-T. B. (Bliss, 1918) S. lavender blue; F. white. Pale golden beard, and a few purple markings at base of falls. Late. Vigorous and free blooming ................ \$1.00

BALDER-(G \& K) S. yellow-olive, dome shaped; F. dark red-purple. Vigorous in growth and very free blooming ................\$3.00 each

94 BALLERINE-T. B. (Vilmorin, 1920) S. light violet blue; F. deep violet blue. Large, well formed flower on tall, branching stems. Vigorous. 42 in.. $\$ 1.00$

77 BLACK KNIGHT-(Perry) S. dark violet; F. purple ................... \$1.00

83 BLUE BIRD-(Bliss, 1919) Bright of good form and medium size with spreading falls.......50 each

67 BLUE BOY-I. B. (Foster, 1913) S. and F. rich purple, with blue beard. Early. 24 inches..........25c

91 BRANDYWINE-T. B. (Farr, 1920) S. and F. pale silver blue, orange beard. Very fine and very scarce .................... \$1.00

79 CAMELOT-T. B. (Bliss, 1918) White with pale violet edge. Tall and branching. Plicata type. 48

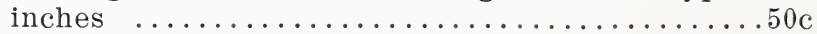

CANOPUS-(Bliss) Greyish lavender standards, rich purple falls with white markings near a clear yellow beard. Large flowers, on 30 inch stalk. Very fine .................... \$3.00

CAPORAL-T. B. (Bliss, 1919) Bright, reddish-violet self. Large flowers. Vigorous, free bloomer...75c

75 CAPRICE-T. B. (Vilmorin, 1904) S. rozy red; F. deeper rosy-red, shading toward white at centers. A fine red-purple. 24 inches..........40c; $\$ 3.50$ per doz.

CAROLINE E. STRINGER-(Sass, 1924) A light, pale pink of good size and form. Medium height and vigorous in growth and bloom. Will rate high

$\$ 4.00$ each 
CARTHUSIAN-T. B. (Marshall, 1906) S. clear lavender blue; F. deeper blue................

89 CATERINA-T. B. (Foster, 1909) Lavender, blue and lilac. Larger flower, taller and more graceful than Pallida Dalmatica, which it resembles. 48 in...50c

CAVALIER-T. B. (Cleveland, 1920) S. clear blue; F. deep velvet purple, white at throat, with blue veins. Strong and upright ..................

CECIL MINTURN-T. B. (Farr, 1922) S. and F. uniform shade of cattleya rose, with light beard. Large dome shaped flower. Nearly a true pink. 30 inches .......................

71 CELESTE-T. B. (Lemon, 1855-58) S. and F. pale azure-blue. Large flower. 30 inches. .25c; $\$ 2.50$ doz.

86 CLUNY-T. B. (Vilmorin, 1930) S. soft lilac blue; F. slightly darker, with brown veinings at base. Large flower on a tall stem. Very fine. Early. 39 inches ...........................

80 COL. CANDELOT-T. B. (Millet, 1907) S. smoky copper; F. brick-red. Large and free flowering Squalens. 36 inches ...................

CONQUISTADOR-T. B. (Mohr) S. and F. deep mauve to light violet. Very tall, well over five feet and large flower ................. \$5.00

CORA-T. B. (Millet, 1914) S. lavender, F. dark helio-

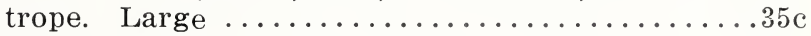

71 CORDELIA-T. B. (Park, 1873?) S. rosy-lilac; F. velvety crimson edged with rose. Late. 24 in......50 c

77 CORDON BLEU-T. B. (Sturtevant, 1921) S. and F. satiny Dauphin's violet, drooping and rounded falls; white, orange tipped beard. 30 inches.......50c

83 CORRIDA-T. B. (Millet, 1914) S. and F. analine blue, slightly darker than Celeste. Neat flower, but not large. 36 inches ................ \$1.00

84 CRETONNE-T. B. (Bliss, 1919) S. pale bronze-purple; F. rich maroon with orange beard. Not a large flower but very distinct. 36 inches........75c

89 CRIMSON KING-T. B., S. and F. rich claret-purple. Free bloomer. Vigorous. 28 inches.........20c

87 CRUSADER-T. B. (Foster, 1913) S. light blue; F. deep violet blue with orange yellow beard. Fine color and form. Slow grower. 42 inches......75c

80 DALILA-T. B. (Denis, 1914) S. pale flesh-white; F. rich red-purple, with yellow beard. 24 inches....50c

DAPHNE-T. B. (Bliss, 1920) S. pure white; F. rich purple with distinct white margin. Very clear colors and very effective.............\$1.50

78 DAWN-T. B. (Yeld, 1911) S. and F. sulphur yellow, veined bronze at the throat. Free bloomer; vigorous. 27 inches ...................50c 
83 DEJAZET-T. B. (Vilmorin, 1914) S. bronze rose with golden sheen; $\mathrm{F}$. reddish violet, shaded brown. Large, broad flower. Rather dwarf. Late. 24 inches ...........................

82 DELICATISSIMA-T. B. (Millet, 1914) Delicate lilac self. Large, on tall, branched stalk. 36 inches. .\$1.00

69 DEMI-DEUIL-T. B. (Denis, 1912) S. amber yellow, heavily veined and dotted deep purple; F. white, veined and dotted light purple. Attracts instant attention in the garden...............50 c

76 DILKUSH-M. B. (Foster, 1909) S. violet, with rich netted veinings; F. reddish purple with black throat and yellow beard. Large, rounded flower. Oncocyclus and Pogoniris hybrid. 27 inches..... \$1.00

83 DIMITY-T. B. (Bliss, 1919) S. and F. pure white, with faint blue veinings on margins. Pallida form. 36

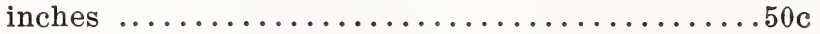

94 DOMINION-T. B. (Bliss, 1917) S. light bluish violet; F. rich indigo-purple, with distinct orange beard. Wonderful in size, color and texture, but rather tender and slow growing. 30 inches........\$10.00

81 DORA LANGDON-T. B. (Bliss, 1918) S. creamy buff, flushed pink at center; F. magenta shading to pale rose-purple at edges. Orange beard. Vigorous. 30 inches ............................

76 DOROTHEA-I. B. (Caparne, 1901) Lilac blue, tinged with white; S. flat like a Japanese iris ........35c

DOROTHEA K. WILLIAMSON-Hex. (Williamson, 1918) Velvety reddish-purple. A beautiful, beardless iris requiring a rich moist soil. Very desirable.. $\$ 1.00$

75 DR. ANDRIST-T. B. (Fryer, 1919) S. bronze yellow; F. velvety maroon, with bronze edging. Very late Yellow beard. Fragrant ..............50

74 DR. BERNICE-T. B. (1867) S. Coppery-bronze; F. velvety crimson. Large, handsome flower. Late. 30

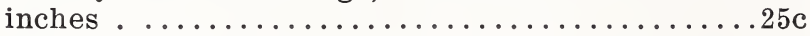

85 DREAM-T. B. (Sturtevant, 1918) Lilac self; S. arching, deeply notched at tips; F. drooping with slight flare. As popular in the Iris collection as Lady Alexandra Duff is among the Peonies. Vigorous.

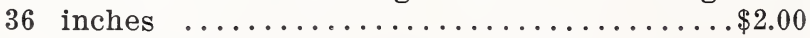

ECKESACHS-T. B. (G \& K, 1920) S. delicate lavender blue; F. bright purple-violet of fine form. Early and free blooming. Vigorous. 36 inches......75c

86 EDOUARD MICHEL-T. B. (Verdier, 1904) S. rosyviolet; F. deeper violet. Large and beautiful. 36

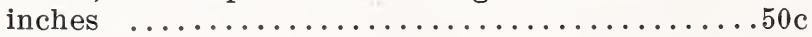

78 ELDORADO-T. B. (Vilmorin, 1910) S. yellow-bronze, shaded with heliotrope; F. violet-purple with bronze yellow tints. 30 inches...............50

ELIZABETH HAMILTON-T. B. (Braillier). A blue and white plicata of good size and substance. Medium height $\ldots \ldots \ldots \ldots \ldots \ldots \ldots \ldots \ldots \ldots \ldots . \ldots \ldots$ 
FAIRY-T. B. (Kennicott, 1905) White, delicately bordered with pale blue. Tall and fragrant. A great favorite. 40 inches .........25c; $\$ 2.50$ doz.

FIRMAMENT-I. B. (Groschner) S. clear light blue; F. bright blue, with purple in center and bright orange beard. Prolific and vigorous.......\$1.00

FLAMMENSCHWERT-T. B. (G. \& K.) S. and styles, empire yellow; F. velvety brown with yellow border. Vigorous. Well branched. Free flowering. $30-36$ inches . ................. \$1.00

76 FLORENTINA-T. B. White with faint lavender tints. Very early and fragrant. 24 inches..25c; $\$ 2.50 \mathrm{doz}$.

FOLKWANG- $(G$ \& K) F. claret with conspicuous veinings of brownish yellow and light edging. S. light rose-pink. Very odd and conspicuous....\$3.00

FORSETE- $(G$ \& $K)$ A dark lavender flower of Pallida Dalmatica size, borne on an unusually branching stem. 36 to 48 inches in height.......\$3.00

70 FRANCINA-T. B. (Bliss, 1920) Pale lavender-white; S. spotted and veined reddish-purple; F. edged reddish-purple. 36 inches ............. $\$ 1.00$

FUERSTIN LONYAY-T. B. (G. \& K., 1920) S. violetlilac; $F$. wine-red. Often has 6 to 8 blooms on a stalk. Vigorous ....................

GEO. J. TRIBOLET-(Williamson, 1926) A magnificent large coppery purple flower on 40 inch stems. S. Nigrosin violet; $F$. velvety blackish purple, the flower suffused purple .............. \$5.00

89 GEORGIA-T. B. (Farr, 1920) S. and F. uniform shade of soft cattleya rose. Bright orange beard. A delicate and beautiful variety. Vigorous. $30-36$ in.. $\$ 1.00$

GLAMOUR-(Bliss) Gray and purple.......\$15.00

GLITTER-T. B. (Bliss, 1919) S. yellow; F. chestnutbrown, with yellow edge. Early. Free flowering. Very bright in mass. 27 inches...........50c

68 GLORY OF READING-T. B. (Farr, 1909) S. deep blue; $F$. royal purple. Large handsome flower. Fragrant ..........................

GLOWING EMBERS-T. B. (Sturtevant, 1923) S. violet flushed; F. rich dahlia purple, with vivid orange beard. Yellow in throat. Large flowers of fine substance. Tall, well branched. The first of a series. 42 inches ............... \$7.00

71 GOV. HUGHES-T. B. (Fryer, 1919) S. deep violet, tinged red; $F$. deeper shade. Large orange beard. a clump of this, showing a mixture of violet and orange, is very striking. 27 inches........50c

85 GREVIN-T. B. (Vilmorin, 1920) Deep purple, with standards shaded with yellow. Strong stems. Large flowers ............................

71 HAMADAN-M. B. (Foster, 1909) S. light violet; 5 . dark purple violet, horizontal and with broad black beard. Oncocylus and Pogoniris hybrid......\$2.00 
HARRIET PRESBY-T. B. (Presby, 1922) Petunia violet, with orange tipt beard. Vigorous, high, well branched stalk. 48 inches ...........\$3.00

74 HAUTEFEUILLE-T. B. (Denis, 1910) Dark violetpurple. Very tall and greatly admired. 36 in...\$1.00

HELENE KOEHLER-(Koehler, 1924) A Lohengren seedling of pink tone. S. delicate pink; F. delicate pink with slightly darker veining. Vigorous growth, with 32 inch stems ..............\$5.00

73 HER MAJESTY-(Perry) S. lovely rose pink; F. same color, veined darker. Still one of the best of the

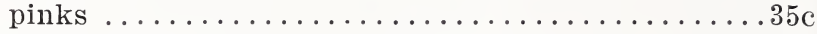

HERMIONE-T. B. (Hort, 1920) S. bright blue purple, F. rich red purple. Large and fragrant.....\$3.00

HESPERIA (Williamson, 1926) S. light drab; F. solid blackish, red purple, flushed amber. Large flowers and a strong grower. 36 inches.........\$3.00

IGOUF-(Millet) Blue and violet........\$1.00

ILSAN-T. B. (G. \& K., 1920) S. light lilac rose; F. dark wine red. Free bloomer. Late, 30 in... \$ $\$ 1.00$

IMPERATOR-(Cayeux, 1921) S. clear violet, shaded fawn; F. reddish violet, reticulated red-brown at throat. A very large flower, on good stiff stems about 36 inches tall. Vigorous in growth...\$5.00

INCA-T. B. (Farr, 1922) S. clear, deep saffron yellow; F. velvety dark plum, edged with gold. Touches of gold in center and at base. Late. 18 in....75c

77 INGEBORG-I. B. (G. \& K., 1908) Pure white, large flowers. Free bloomer; vigorous..........30c

79 IRIS KING-T. B. (G. \& K., 1907) S. old gold; F. deep maroon, bordered yellow. Large, fine and brilliant. 24 inches ........................

86 ISOLINE-T. B. (Vilmorin, 1904) S. lilac-pink; $\mathbf{N}$. purplish old rose, golden at the throat and with orange beard. Very conspicious, and among the best. 36 inches .............50c; 3 for $\$ 1.00$

80 JACQUISIANA-T. B. (Lemon, 1840) S. bright coppery-crimson; F. rich maroon. Distinct and beautiful. 36 inches ..............50c; $\$ 5.00$ doz.

70 JAMES BOYD-T. B. (Farr, 1915) S. immense, of clear light blue, broad and incurved, forming a high dome shaped center; F. dark violet, tipped and edged lighter. 32 inches..............50c

84 J. B. DUMAS-T. B. (Denis, 1917) Rose pink. Large and fine. Ricardi hybrid ...........\$5.00

78 JEANNE D'ARC-T. B. (Verdier, 1907) S. and F. white, frilled with lilac blue. 30 in..35c; $\$ 3.50$ doz.

JUBILEE-T. B. (Sass, 1923) S. peach yellow; F. flaring; white, dotted and striped maroon on haft. Deep yellow beard. Ruffled and of heavy texture. 30 inches ................... \$8.00

81 JUNIA.TA-T. B. (Farr, 1909) Clear blue, S. arching and F. flaring. Large and fragrant. 42 inches. $35 \mathrm{c} ; \$ 3.50 \mathrm{doz}$. 
KALOS-T. B. (Sass, 1924) S. and F. white, lightly veined and flecked pale rose. Slightly ruffled. Standards full and arching, prominent yellow beard. This attracted so much attention in our gardens that we bought the entire stock with the privilege of introducing it...........\$3.00

84 KASHMIR WHITE-T. B. (Foster, 1913) S. pure white; F. pure white, faintly flushed with grey-blue, flaring. Must have good drainage. 42 inches..\$1.00

81 KATHRYN FRYER-T. B. (Fryer, 1917) S. lurge, bright yellow; $F$. velvety maroon, veined white in center, yellow at base, orange beard. Fragrant. 32 inches ..................... \$1.00

78 KNYSNA-T. B. (Bliss, 1917) S. clear yellow; F. deep velvety brown. Well branched and free blooming. Vigorous. 32 inches ................ $75 \mathrm{c}$

78 KOCHI-T. B. Rich claret-purple. Early. 24 inches. $35 \mathrm{c} ; \$ 3.50 \mathrm{doz}$.

LADY BING-T. B. (Bliss, 1922) S. and F. clear lavender, suffused with rose. A fine new flower. $\$ 10.00$

85 LADY FOSTER-T. B. (Foster, 1913) S. pale blue; F. pale bluish violet. Large flowers of fine texture. Fragrant. 40 inches ............... \$1.00

96 LENT A WILLIAMSON-T. B. (Williamson, 1918) S. soft violet, broad and erect; $F$. velvety royal purple, with yellow beard. Large, free blooming and vigorous. 42 inches ....................

LEPINOUX-T. B. (Millet, 1923) S. lobelia blue, F. violet with bronze veins, white at base. Large and tall .........................\$3.00

90 LEVERRIER-T. B. (Denis, 1917) S. mauve; F. pansy violet, hanging straight. Tall, branching stems. Fragrant. Vigorous. One of the best. 42 in...\$2.00

82 LOHENGREN-T. B. (G. \& K., 1910) S. light mauve; F. Chinese violet fading to white toward center. Orange beard. Vigorous. High, well branched stalk.

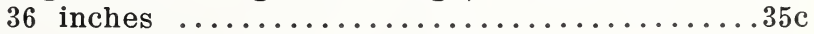

LOIE FULLER-(Vaughn) Pure yellow .........35c

LONGIPETALA-S. and F. soft porcelain blue. Leaves tall and straight. Every stem produces seed. 36

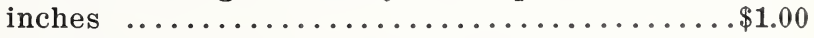

91 LORD OF JUNE-T. B. (Yeld, 1911) S. lavender blue; F. rich violet blue. Large flower. Strong and vigorous. Fragrant. 40 inches...........\$1.00

78 LORELEY-T. B. (G. \& K., 1909) S. light yellow; F. ultramarine blue, bordered with cream. 30 inches. $25 \mathrm{c} ; \$ 2.50 \mathrm{doz}$.

74 MADAME CHEREAU-T. B. (Lemon, 1844) White, beautifully frilled with blue. 36 in..20c; $\$ 2.00 \mathrm{doz}$.

85 MADAME CHOBAUT-T. B. (Denis, 1916) S. golden yellow, heavily veined with deep wine-purple; $F$. pale olive buff slightly edged with deep wine purple. Yellow beard, brown tipped. Fragrant. Vigorous.

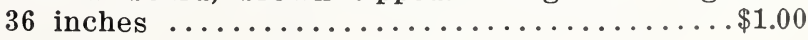


86 MADAME DURRAND-T. B. (Denis, 1912) S. and F. buff and creamy yellow, suffused with mauve. Large and tall .......................... $\$ 30$

79 MADY CARRIERE-T. B. (Millet, 19..) S. greyishlavender; F. clear blue, shading to yellow at base. 36 inches ..........................

91 MAGNIFICA-T. B. (Vilmorin, 1920) S. light, violet blue; $F$. dark, reddish violet with brown stripes at base and yellow beard. Very large on tall slender stalk. 48 inches ................ \$1.50

81 MA MIE-T. B. (Cayuex, 1906) White, frilled with pale blue at margin. S. overlapping; F. flaring. 36 inches ...................... $\$ 3.50 \mathrm{coz}$.

71 MARGARET MOOR-T. B. (Bliss, 1918) Reddish lilac self, slightly deeper in falls. Tall. 33 in...\$1.00

MARIPOSA-T. B. (Mohr) S. and F. pale porcelain blue, flaked with red purple. Medium size. Late.. $\$ 1.00$

87 MARSH-MARIGOLD-T. B. (Bliss, 1919) S. pale golden yellow; F. purple brown, with a conspicuous yellow edge. 27 inches $\ldots \ldots \ldots \ldots \ldots \ldots \ldots 1.00$

78 MARY GARDEN-T. B. (Farr, 1913) S. pale yellow, flushed pale lavender; F. Cream white, minutely dotted and veined with maroon. 30 inches.....50 c

MARY ORTH-(Farr) S. light violet; F. dark blue

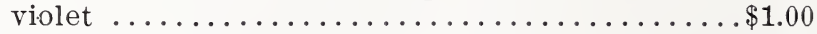

76 MAUVINE-T. B., S. mauve; F. darker mauve. Orange beard. Tall and free flowering. Vigorous, 36

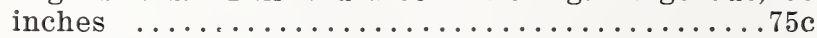

MAY ROSE-T. B. (Cleveland, 1920) S. and F. bright but soft rose pink. Good size and substance. 30

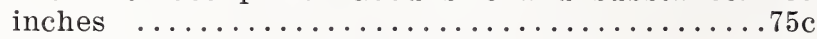

85 MEDRANO-T. B. (Vilmorin, 1920) S. and F. beaut1ful dark, smoky red. Large. Medium early. 26

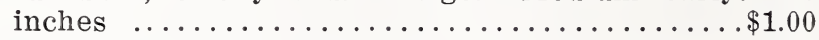

83 MERCEDES-T. B. (Verdier, 1905) S. purplish-lilac, shaded bronze; F. white, dotted and penciled with purple. Orange beard. 30 inches........\$1.00

MIDWEST-T. B. (SasS, 1922) White, heavily bordered and spotted Matthew's purple; S. and flaring falls are ruffled. Vigorous and well branched. 30

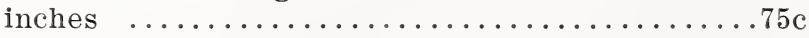

MILDRED PRESBY-(Farr, 1923) White standards; falls of rich, dark, pansy violet. A much improved Rhein Nixie, wonderfully effective in the garden

$\$ 3.00$

MIRANDA-T. B. (Hort, 1919) Clear violet blue. Orange beard. Early. Fine form. Stout stems. 36 inches ................... \$1.00

76 MISS WILLMOTT-T. B. (Foster, 1910) White, slightly tinted lavender. Petals of stout leathery texture. Falls at right angles. 40 inches..........50

MISTRESS FORD-T. B. (Hort, 1921) Reddish pur. ple. S. heavy and upright. Large; fine texture and vigorous. New and rare...........\$3.00 
75 MITHRAS-T. B. (G. \& K., 1910) S. light yellow; F. brilliant wine red, bordered deep yellow. 30 in.. $\$ 1.00$

87 MLLE. SCHWARTZ-(Denis) S. \& F. uniform lavender pink, with yellow beard. Large flowers on tall, branching stems. The best of its type and color...\$1.50

90 MOA-T. B. (Bliss, 1919) S. pure violet, broad, arching, almost circular; F. deep violet purple, smooth and flat. Very large flowers, of great substance. Tall and branched. 42 inches..........\$20.00

85 MOLIERE-T. B. (Vilmorin, 1920) S. rich violet; F. darker velvety violet, veined brown at haft. Yellow beard and large dark yellow styles. 28 inches. $\$ 1.00$

MONSIGNOR-T. B. (Vilmorin, 1907) S. rich violet; F. velvety crimson-purple with deep veinings, except for wide margin, of deeper purple. 26 inches. $25 \mathrm{c} ; \$ 2.50 \mathrm{doz}$.

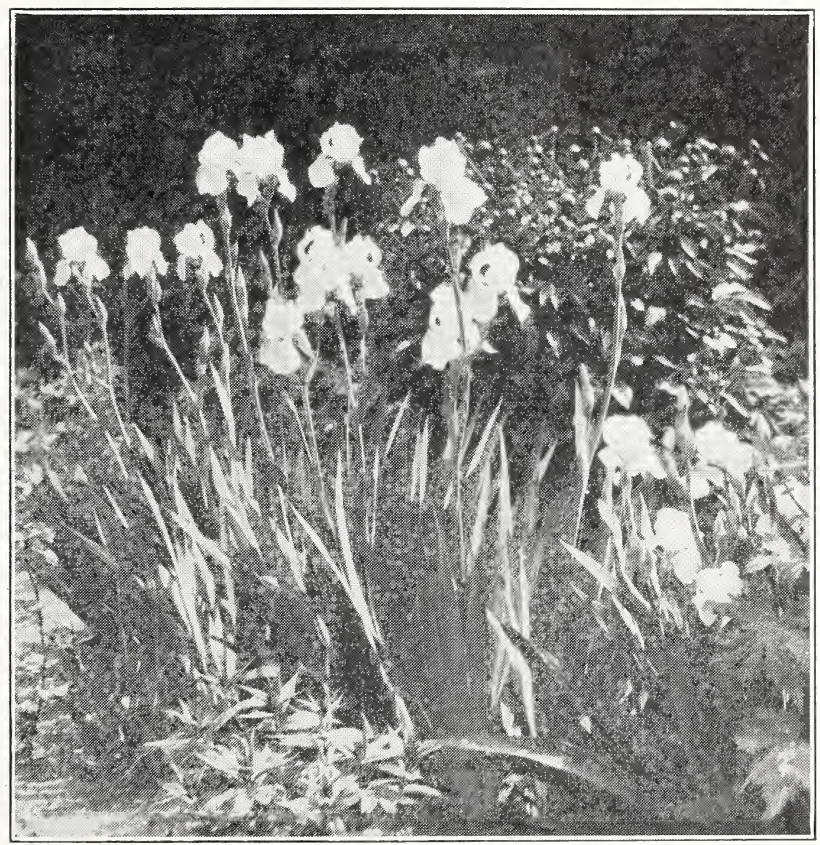

Mother of Pearl with White Knight Below

MOTHER OF PEARL-T. B. (Sturtevant, 1921) Self colored, Mother of Pearl tints. Tall, vigorous and a free bloomer. Fragrant. 40-48 inches.....\$1.00

76 MRS. ALAN GRAY-T. B. (Foster, 1909) S. and F. delicate rose mauve. Free bloomer. Vigorous. 30

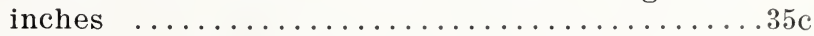

73 MRS. A. M. BRAND-T. B. (Fryer, 1919) S. white; F. white, slightly veined violet. Bright orange beard. Large flower, erect and fragrant. 30 inches...50c

70 MRS. KINGSCOTE-T. B. (Perry, 1911) S. silvery rose; F. rose pink, with prominent yellow beard. Pallida type ..................\$1.00 
68 MRS. NEUBRONNER-T. B. (Ware, 189..) Light cadmium yellow. 24 inches................

MRS. WALTER BREWSTER-T. B. (Vilmorin, 1922) S. lavender blue; F. analine blue, with some brown pencilings. Large flower. Vigorous. 40 in.... $\$ 1.00$

71 NAUSHON-T. B. (Sturtevant, 1920) Mauve and pansy violet. Flower has rather a pointed top, and broad ruffled segments, of slightly craped texture. Well branched and vigorous. 30 inches.......50c

81 NEPTUNE-T. B. (Yeld, 1916) S. bright pale blue; F. dark rich purple blue. Wide spreading falls. Large. 42 inches..............50 c

79 OCHRACEA-T. B. (Denis, 1919) S. variegated yellow, long and narrow; F. yellow tinged with purple. Very distinct and effective. Late. Vigorous. 24

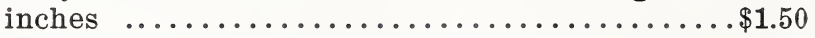

87 OPERA-T. B. (Vilmorin, 1916) S. reddish lilac; F. dark, velvety purple violet. Early. Vigorous. Greatly admired. 32 inches ............\$1.00

79 OPORTO-T. B. (Yeld, 1911) Red purple. Orange beard. Large. Early. Vigorous. 36 inches....50c

ORCHID-T. B. (Shull, 1922) Siena yellow, flushed purple ..................... \$1.00

ORIENTALIS-Siberian. White .........25c

ORIENTALIS-Siberian. Blue ..........25c

78 ORIFLAMME-T. B. (Vilmorin, 1904) S. dark laveuder; F. rich violet purple. Very large, handsome flowers. Variable growth. 30 inches........50c

88 PALLIDA DALMATICA-T. B., S. lavender; F. clear deep lavender. Large and extra fine. 42 inches..35c

80 PALLIDA SPECIOSA-T. B., S. dark, silver lavender; F. shaded light purple. 42 inches..........50c

81 PARC DE NEUILLY-T. B. (Verdier, 1910) Rich plum purple; S. arching and ruffled; F. drooping. Vigorous. 30 inches ....................

79 PARISIANA-T. B. (Vilmorin, 1911) S. white ground, dotted and shaded lilac purple; F. white frilled lilac. Large. 30 inches .......................

75 PARVAR-M. B. (Foster, 1909) Deep blackish-violet with bronze tints. Rich and distinct. Ococylus and Pogoniris hybrid .............. \$2.00

75 PAULINE-T. B. (Farr, 1913) Large, rich pansy violet; deep orange beard. 36 inches.........50c

78 PERFECTION-T. B. (Barr, 1880) S. light lavender; F. velvety black-violet. Orange beard. Striking. 38 inches .........................

89 PHYLLIS BLISS-T. B. (Bliss, 1919) S. and F. pale rosy lavender. Large flowers of fine form. New and rare. Well branched. Vigorous. 36 in.....\$2.00

PINK P'ROGRESSION-(Champ, 192-) A tall pink self, large and of good form. A very distinctive

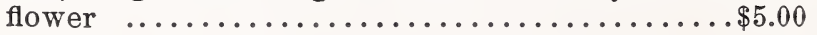


77 POCAHONTAS-T. B. (Farr, 1915) S. pure white, faintly bordered blue; F. pure white, and frilled. large, orchid type. 28 inches...........50c

PONT A MOUSSON-T. B. (Millet, 1919) S. pale, bronze yellow, shot with lavender; F. rich purple, shading to white. Tall and stately..........75c

95 PRINCESS BEATRICE-Tall, light lavender.....75c

PRINCESS VICTORIA LOUISE-(G \& K) Yellow and

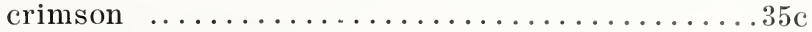

PROF. A. SEELIGER-(Koehler, 1923) A deep winered with 38 inch stems. "An improved Ed. Michel." Very distinct ................ \$2.00

83 PROSPER LAUGHIER-T. B. (Verdier, 1914) S. light bronze red; $\mathbf{F}$. velvety ruby purple, with orange beard. Large flower. 36 inches...........35c

PROSPERO-T. B. (Yield, 1920) S. pale lavender, flushed lavender at base; $F$. deep red purple, shading lighter at margins. Haft heavily veined with brown. Deep orange beard. Large flowers, fine shape. Vigorous. One of the best. 48 inches. $\$ 1.00$

PUMILA-Florida. Yellow ...............

PUMILA-Orange Queen. Yellow .........25c

PUMILA-Prairie Beauty. Golden yellow ......25c

PUMILA-Royal Purple. Large, rich purple ....50c

84 QUAKER LADY-T. B. (Farr, 1909) S. smoky lavender, shaded yellow; F. ageratum blue and old gold, drooping. Orange beard and yellow stigmas. Strong grower and very attractive. $30 \mathrm{in} . .40 \mathrm{c} ; \$ 4.00 \mathrm{doz}$.

75 QUEEN ALEXANDRA-T. B. (Barr) S. fawn, shot with lilac; F. lilac, reticulated bronze at base. Yellow beard. 30 inches ..........35c; $\$ 3.50$ doz.

90 QUEEN CATERINA-T. B. (Sturtevant, 1917) Pale lavender violet. S. domed; $\mathrm{F}$. drooping and rounded, with yellow beard. Haft veined with bronze. Large flower. Vigorous. One of Miss Sturtevant's best. 3 inches ................. \$1.25

74 QUEEN OF MAY-T. B. (Salter, 1859) S. and F. soft rosy-violet. An old favorite. 32 inches.......25c

75 RACHEL FOX-T. B. (Jacobs, 1920) S. pale gold, arching and slightly cupped; F. golden yellow, with blue flush, drooping. Free flowering. Vigorous. 32 inches ..................... \$1.00

81 RAFFET-T. B. (Vilmorin, 1920) Uniform dark blue, with falls striped with white at base. Yellow beard. Late. 26 inches ...............\$1.00

78 RED CLOUD-T. B. (Farr, 1913) S. rosy-lavenderbronze; F. velvety maroon crimson, reticulated yellow, old gold stigmas. 26 inches......... $\$ 1.00$

RED RIDING HOOD-(Koehler, 1922) One of the new "reds." S. reddish lavender; F. dark red-purple reticulated brown at the base. A strong grower, with 32 inch stems. Of decided merit......\$2.50 
RENE CAYEUX-(Cayeux, 1924) Very large flowers of distinct coloring. S. white, shaded lilac; F. reddish crimson violet, reticulated white at the throat.

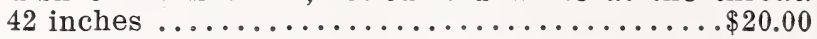

87 REVERIE-T. B. (Sturtevant, 1920) S. light wine lilac, shading to Colonial buff, with a narrow border of pink; F. solid Helebore red with wide white haft. Of the finest substance, form and habit. (From Originator's description). 36 inches ........\$8.00

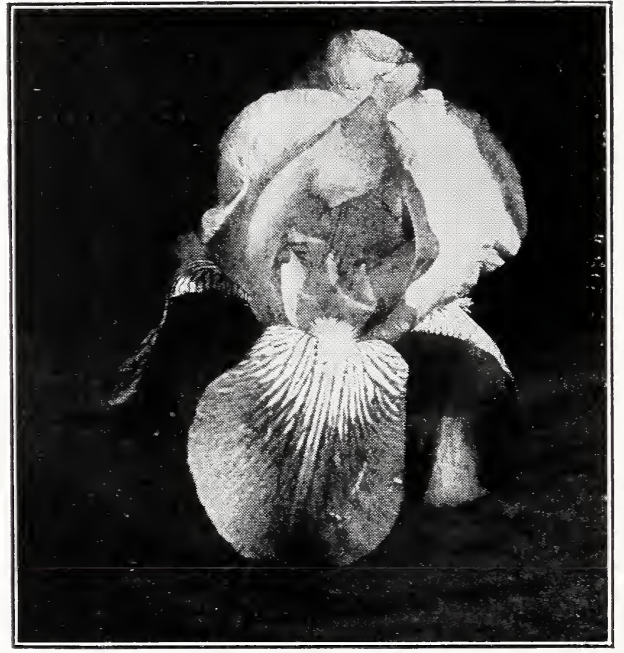

Reverie

REGAN-(Hort) Clear, dark, blue-purple self. Falls slightly deeper. Deep yellow beard. 4 inch flower. With us an excellent and outstanding variety.. $\$ 1.00$

RHEINGAUPERLE- $(\mathrm{G} \& \mathrm{~K})$ Both standards and falls of a soft rose pink, something like Susan Bliss. The standards are very broad and arch into a dome shaped head. Distinct and beautiful........\$3.00

84 RHEIN NIXIE-T. B. (G. \& K., 1910) S. white; F. violet blue, white edge. Tall, large and fine. 36

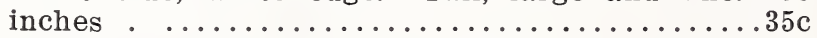

RHEINTRAUBE-T. B. (G. \& K., 1917) S. clear lavender blue; F. purple violet. Vigorous; well branched. 36 inches .................... \$1.00

78 RICARDI FONCE-T. B. (Denis, 1916) Bright violet, deepening in falls to Bradley's violet. Large flowerea on tall, branched stems .........\$1.00

74 RICHARD I-T. B. (Dykes, 1914) S. white; F. deep velvety purple. Large flower, dwarf and slow growing. Late. 20 inches..............\$1.50

76 RING DOVE-T. B. (Foster, 1913) S. pale violet; F. lavender violet with distinctly darker ring at the throat. Free flowering. 36 inches........ $\$ 1.00$

83 RODNEY-T. B. (Bliss, 1919) Dark violet blue self. S. domed; F. drooping. Free flowering. Branched. Vigorous. 36 inches $; ; ; ; \ldots \ldots \ldots \ldots \ldots \ldots \ldots . . \ldots 1.00$ 
80 ROMEO-T. B. (Millet, 1912) S. lemon yellow; F. lilac center, shading to velvety maroon violet at edges. Rigid flower of fair size. Moderate growth. 30 inches ................... \$1.00

69 ROSE UNIQUE-T. B. (Farr, 1910) Bright violet rose. Rare in color. Early. 26 inches...........50c

82 ROSEWAY-T. B. (Bliss, 1919) Phlox-purple Pallida, with broad orange beard. Early. Branched. 38

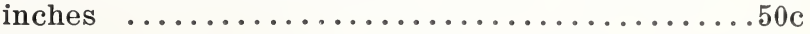

ROTORUA-T. B. (Bliss, 1921) S. pale fresh lavenderblue; F. same shade suffused with deeper blue at center. Very fine ............... \$1.00

SAN GABRIEL-(Dean) A clear pale mauve flower of large size, carried on a well branched stem that measures 44 to 48 inches. Very early....... $\$ 3.50$

SAVIGNIAN-T. B. (Millet, 19..) S. violet; F. pansy violet. Rich coloring; medium flower. 30 in... $\$ 1.00$

SCHWANHILD-T. B. (G. \& K., 1920) Similar to Trautlieb, but a clearer pink. Free flowering..\$2.00

SEMINOLE-T. B. (Farr, 1920) S. Soft velvet rose; F. rich velvet crimson. Orange beard. Large and

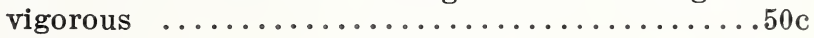

77 SHALIMAR-T. B. (Foster, 1916) Rich violet purple. Well branched and of fine size. A Trojana seedling. 40 inches ...................

87 SHEKINAH-T. B. (Sturtevant, 1918) Pale lemon yellow, deepening through center. Orange yellow beard. The first clear yellow of Pallida growth. 36 inches ................... \$1.00

SHELFORD GIANT-Spuria. (Foster, ...) S. creamy yellow; F. cream with broad orange blotch. Vigorous and handsome. 48-56 inches.........\$3.00

81 SHERBERT-T. B. (Sturtevant, 1918) S. ecru drab, deepening to purple; F. dahlia purple, shading lighter at edges. Very effective. 44 inches........75c

76 SHERWIN WRIGHT-T. B. (Kohankie, 1915) S. and F. golden yellow. Vigorous and free flowering. 28 inches . .........................

82 SHUSHAN-M. B. (Foster, 1913) S. purplish grey; F. deep crimson maroon, almost black, bright yellow at the haft. Free flowering. Very dainty. Oncocyclus-Pogoniris hybrid ............\$2.00

SILVER RIBBON-(Williamson, 1926) A deep pink, self colored flower, with a pale band down the center of each fall. Large flowers of heavy texture. 30

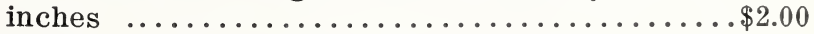

SOLANA-T. B. (Shull, 1921) S. bright yellow; F. Bordeaux red, or claret ............\$1.50

93 SOUVENIR DE MME. GAUDICHAU-T. B. (Millet, 1914) S. deep analine blue; F. deep purple violet, drooping. Very warm colors. Widely branching stalk. Vigorous. 40 inches............\$1.25 
SPUREA AUREA-A beautiful species from the Himalayas. Large, deep, golden yellow flowers. $4 \mathrm{ft}$. June and July ...............\$1.00

SPUREA-Monspur, (Foster) Violet blue.......50c

SPUREA, OCHROLEUCA-White, with orange yellow

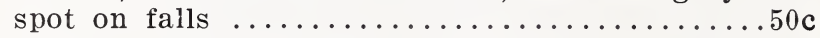

STEEPWAY-T. B. (Scott, 1922) S. fawn, snading to reddish fawn at edges, round; $F$. rosy mauve, shading to opalescent blue at base, with brown markings and orange beard. Vigorous. 36 inches.....\$1.00

80 SUNSHINE-T. B. (Yeld, 1911) Brilliant self colored yellow. Effective when massed. 26 inches... $\$ 2.00$

SUSAN BLISS-(T. B. (Bliss, 1922) Rich, light pink. Fine shape, tall and free blooming. One of the

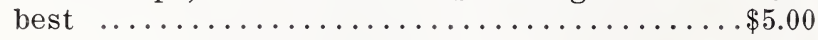

SWAZI-(Bliss, 1922) A very dark and rich Dominion seedling. S. bluish violet; $F$. velvety blackish violet. Very vigorous in growth with superb flowers on a $36-40$ inch stem ...............\$15.00

85 SWEET LAVENDER-T. B. (Bliss, 1919) S. pale lavender; F. deeper rose-lavender, broad and horizontal. Large, perfect formed flowers. Strong, branching steams. 38 inches ............\$1.75

TAFFETA-T. B. (Cleveland, 1920) S. Pale brown tone, shaded with blue and gold, changing to soft fawn color; F. fawn, shot with blue. Large and tall . ................... \$1.00

74 TAMAR-T. B. (Bliss, 1920) S. reddish lilac; F. veined and suffused deeper reddish lilac, semi-spreading. Large and showy. Vigorous. 42 inches.. $\$ 1.00$

74 TAMERLAN-T. B. (Vilmorin, 1904) S. purple-violet; F. deep purple-violet. Free flowering. Orange beard. 30 inches .....................

TENEBRAE-(Bliss, 1922) A richly colored Dominion seedling. S. deep violet purple, with dark maroonpurple shades in center. F. very dark black-purple with some brown on haft. A very fine and beautiful flower. Medium height and slow growing. $\$ 10.00$

TITAN-T. B. (Bliss, 1919) S. light violet blue, conical; F. violet purple, deeper in center, reticulated purple on white at haft. Vigorous. Free bloomer. The Giant of the Dominion race. 3 inches... \$5.00

TRIANON-T. B. (Vilmorin, 1921) S. pale buff, with a faint rose cast; F. pale buff, deepening to lavender. Orange beard. Free flowering and vigor-

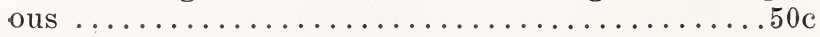

81 TROJANA-T. B., S. pale blue; F. rich purple blue, long. Large, handsome flowers. Late. Branched. 36 -40 inches ......................

74 TUNISIE-T. B. (Millet, 1919) S. slate-violet, with slight yellow cast; F. velvety mauve-lilac. A tall Squalens ..................... 
TURCO-T. B. (Vilmorin, 1921) S. bright violet, suffused with buff at base; F. light bluish violet, suffused yellow at base of blades. Prominent pale yellow beard .......................

83 UTE CHIEF-T. B. (Farr, 1920) S. light blue-violet; F. deep royal purple, heavily reticulated. Very large. 36 inches ...................

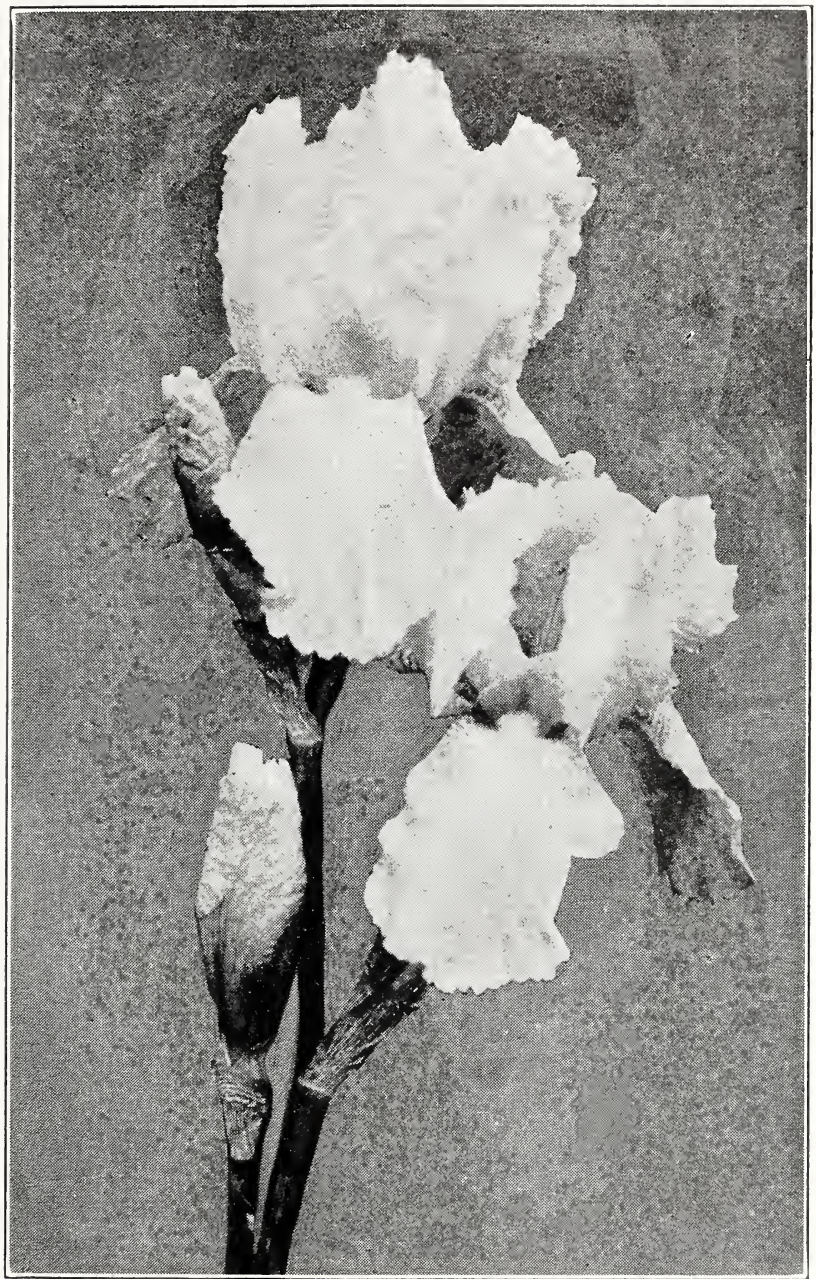

Zua

85 VALERY MAYET-T. B. (Denis, 1912) S. copperyrose; $F$. deep reddish brown. A rare and highly commended French variety. 33 inches........75c

VINCENTIO-T. B. (Hort, 1921) Large purple bi-color, with especially dark falls. Resembles Hermione, though redder in tone. 34 inches........\$5.00 
VINGOLD-(G \& K) Cream standards; falls, deep velvety violet-blue with lighter clear cut margins. An effective color contrast........\$3.00 each

81 VIRGINIA MOORE-T. B. (Shull, 1921) Clear, lemon yellow, of high intensity. Slightly veined the first year. The tallest yellow to date. 33 inches....\$1.00

83 WHITE KNIGHT-T. B. (Saunders, 1916) A pure white self, without any markings. Slightly fragrant. 18 inches ..................

72 WYOMISSING-T. B. (Farr, 1909) S. creamy white suffused delicate soft rose; $F$. deep rose base shading to flesh colored border. Strong and free flowered. Effective in mass. 24 inches.........50c

ZANARDELLE-T. B., S. bright blue; F. somewhat lighter, but.bright blue, horizontal.......\$1.00

ZOUAVE-T. B. (Vilmorin, 1922) S. white, with pale blue tints; upright and arched; F. white, much spotted and reticulated with violet on margins. Vigorous, free flowering ..................

75 ZUA-M. B. (Unknown, introduced and named by Mrs. Crawford) S. and F. white, slightly tinged lilac. Texture like heavy frosted crape, edges are crimped and crinkled. A good multiplier and if not divided too often will have 18 to 20 inch stems, with 5 to 7 immense flowers on each. 18 inches.......\$1.00

85 ZWANENBURG-M. B. (Denis, 1909) S. greyish fawn, reticulated pale violet; $F$. yellow, reticulated pale violet, with yellow beard. Large flowers. Vigorous. Free bloomer, but rather small plants....\$1.00

Our Gaiety assortment of Irises will give you many colors for that long border- $\$ 5.00$ per hundred.

\section{Hardy Perennials}

ANCHUSA ITALICA-Dropmore variety. Tall spikes of deep blue flowers in July and August. 4 to 5 feet

3 for $85 \mathrm{c}$

ANEMONE JAPONICA-Large open flowers from August

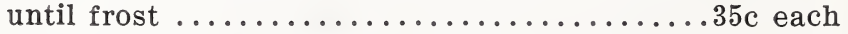

ARABIS ALPINUS-Rock Cress, very early, low and

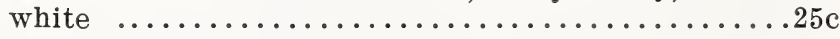

ARMERIA FORMOSA-Cushion pink. Dwarf, compact growing plants with pink flowers..............

ARTEMESIA LACTIFLORA-Old Man; Silvery, fernlike

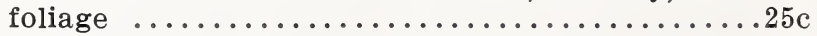

A'RTEMESIA SILVER KING-Silver white stem and

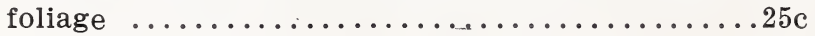

ASTER OR MICHELMAS DAISY-Very showy and free growing. Tall. Late August to October. Light heliotrope, pale lavender blue and deep purple. State color wanted ...................... \$2.50 doz.

BANEBERRY, WHITE-(Actaea alba)-One of our most beautiful native plants with bright clean leaves and clusters of button-shaped white berries with a distinct black spots on each one. Very rare and unusual. $50 \mathrm{c}$ each; three for $\$ 1.25$ 
BLANKET FLOWER-(Gaillardia aristata). Bright colors of the Navajo blankets. Free blooming and attractive. A center cushion of deep maroon is bordered by petals of orange and yellow, sharply circled by crimson, red or maroon............. for $75 \mathrm{c} ; \$ 2.50$ per doz.

BLAZING BEEBALM-(Monarda didyma). Large heads of scarlet flowers shaped like cactus dahlias. $1 \frac{1}{2}$ to 2 feet. Fine for massing; very vigorous......3 for $75 \mathrm{c}$

BLEEDING HEART-(Dicentra spectabilis). An old fashioned favorite. Long racemes of graceful, heartshaped flowers of deep reddish pink. Does well in

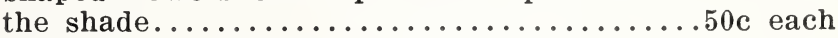

BLEEDING HEART-Western or Plumy (Dicentra formosa). Dwarf growing, with fine cut foliage and a smaller lavender pink flower. Practically an everbloomer............... for $75 \mathrm{c} ; \$ 2.50$ doz.

BOLTONIA ASTEROIDES-Grows about five feet tall and in early autumn is a mass of light lavender daisy-like flowers, with yellow centers. 3 for $75 \mathrm{c} ; \$ 2.50$ per doz.

BOLTONIA LATISQUAMA-A dwarfer variety, about three feet, with pink flowers. 3 for $75 \mathrm{c}$; $\$ 2.50$ per doz.

CHRISTMAS ROSE-(Helleborus nigra) Pretty pure white, single flowers about 9 inches above the low evergreen foliage. Blooms during the winter.50c each

CHRYSANTHEMUM-(C. hortorum). The last flower to bloom in the fall and with its bright colors gives cheer to the "melancholy days." We have some eighteen varieties. The small pompons come in clusters. The largest ones do not exceed three inches in diameter in the garden. We do not have the greenhouse forcing varieties. Colors: White, yellow, pink, red and variegated. Garden grown roots. 3 for $75 \mathrm{c} ; \$ 2 \mathrm{doz}$.

COLUMBINE-(Aquilegia caerulea hyb.) Large flowers of various shades of blue, pink and white, on $1 \frac{1 / 2}{2}$ to 2 foot stems. May and June. Require partial shade. Mixed hybrids.......... 3 for $75 \mathrm{c} ; \$ 2.50$ per doz.

CLOVE or GARDEN PINK-(Dianthus plumarius.) Low glaucous-green foliage, with very fragrant lilac-pink double flowers on 10 to 12 inch spikes. These are the old-fashioned pinks of our grandmother's gardens, blooming profusely in May and June. Excellent for borders. Large garden grown clumps.........50c

COREOPSIS LANCEOLATA-Bright golden yellow daisy form of flower. Grood hardy border plant-blooms from June to October.....3 for $75 \mathrm{c} \ldots \$ 2.50$ per doz.

DAISY, RED-(Chrysanthemum roseum). Bright flowers, with fernlike foliage, in bloom for May 30th., and lasting through June. Single................ for $75 \mathrm{c}$

DAISY-SHASTA-(Chrysanthemum leucanthemum hyb.) Very large single white, to double white. Mixed. 3 for $75 \mathrm{c} ; \$ 2.00 \mathrm{doz}$.

FALSE DRAGONHEAD-(Physostegia virginiana). Erect spikes of pale lavender, tubular flowers, 3 to 4 feet. Likes a shady location. Midsummer......3 for $75 \mathrm{c}$

GYPSOPHYLLA-Baby's Breath. Masses of small white

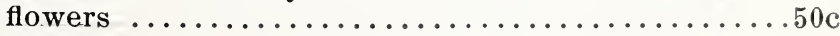


HELIOTROPE, GARDEN-(Valeriana). Showy heads of rose tinted, white flowers in May and June, with delightful heliotrope fragrance.....3 for 50c; $\$ 1.75 \mathrm{doz}$.

HEPATICA TRILOBA-A native spring flowering plant, sometimes called Liverwort or Liverleaf. Flowers are 2 to 4 inches high and are pale blue, lavender and white. Very early, flowers appearing before leaves. $35 \mathrm{c}$ each.

LARKSPUR-(Delphinium). Mixed hybrids of the finer, taller sorts. Very tall spikes. Planted with Madonna lilies, the blue shades are wonderfully effective. 3 for $75 \mathrm{c} ; \$ 2.50 \mathrm{doz}$.

LEMON DAYLILY-(Hemerocallis flava). Pale yellow, very fragrant waxlike bloom. Buds come in clusters on a tall stem and generally one opens each day, thus prolonging the flowering period. May.

3 for $75 \mathrm{c} ; \$ 2.50 \mathrm{doz}$.

LILY OF THE VALLEY-(Convallaria majalis). A favorite perennial, with delicate sprays of drooping, waxlike bells, very fragrant, in early spring. Prefers partial shade and makes a good ground cover. Clumps, 50c; single plants, 75c per doz; $\$ 5.00$ per 100 .

LINUM PERENNE-A small plant, about two feet high, with soft fern like foliage. Each morning from June to September the plant is covered with small clear blue flowers ............ for $75 \mathrm{c} ; \$ 2.50$ per doz.

MEADOWSWEET, EUROPEAN-(Filipendula ulmaria). Low, fern-like foliage, with 2 foot stems bearing lacelike white flowers in May and June. Leaves remain green throughout the winter. Good border plant. 3 for $75 \mathrm{c} ; \$ 2.50$ per doz.

MEADOWSWEET, EUROPEAN, DOUBLE-(Filipendula ulmaria plena). Same as above, except that flowers are very double in clusters..3 for $\$ 1.00 ; \$ 3.50$ per doz.

MOSS PINK-(Phlox subulata). A spreading creeping plant; fine for rock garden or top of stone wall; as all during May it is a perfect blanket of pink flowers. 3 for 50c; $\$ 1.75$ per doz.

PAINTED DAISY-(Chrysanthemum coccineum). Fine hardy border plants, sometimes called Pyrethrum. Excellent for cutting. Mixed colors; pink, lavender, white and red............ for $75 \mathrm{c} ; \$ 2.50 \mathrm{doz}$.

\section{PHLOX:}

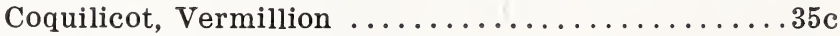

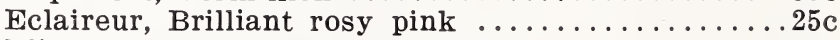

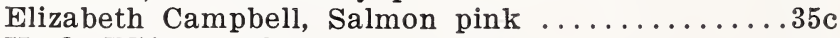

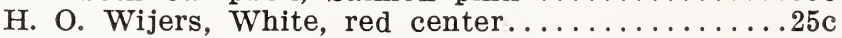

Lord Raleigh, Light, purplish violet.........20c

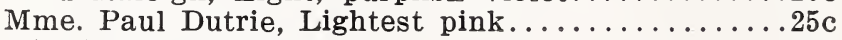

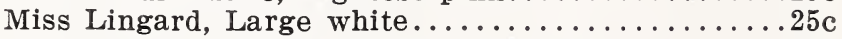

Mrs. Jenkins, Largest trusses of white........25c

Prof. Virchow, Bright orange-scarlet.........25c

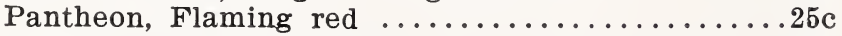

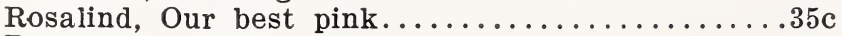

Rosenberg, Large, dark purplish red.........35c

R. P. Struthers, Dark Salmon...........25c

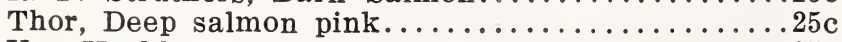

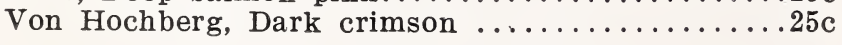


PLANTAINLILY, WHITE-(Hosta plantaginea). White lily-like flowers in terminal racimes. Broad, overlapping leaves. Thrive in shade and excellent for borders.................. for $75 \mathrm{c} ; \$ 2.50 \mathrm{doz}$.

PLANTAINLILY, VARIEGATED-(Hosta). Pale lavender flower, and leaves variegated with white.

3 for $75 \mathrm{c} ; \$ 2.50 \mathrm{doz}$.

POPPY, ORIENTAL-(Papaver orientale). Orange red with black center. Large and showy. It is dormant after blooming but again sends up its thistle-like leaves in September. Can only be transplanted when dormant in August............50 per clump

PURPLE CONE FLOWER-Rudbeckia purpurea) Large crimson-purple flowers, with dark central disc. Sept.

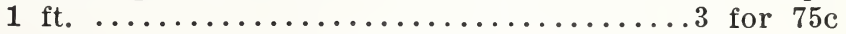

PYRETHRUM ULIGINOSUM-A tall, late flowering daisy, about three feet, with large single white flowers.

3 for $75 \mathrm{c} ; \$ 2.50$ per doz.

ROSE MALLOW-(Hibiscus). Showy ornamental plants for mixed beds or shrubbery borders. Large single flowers of white or pink, with large crimson center. 3 for $75 \mathrm{c} ; \$ 2.50$ per $\mathrm{doz}$.

SHOOTING STAR-(Dodecantheon radicatum) Bright lavender flowers, dartlike and with an almost black eye, borne in umbels on a one-foot stem. Very low foliage. A handsome native flower. April or May..3 for $75 \mathrm{c}$

VIRGINIA BLUEBELL-(Mertensia virginica) Drooping panicles of beautiful blue flowers, changing to lavender pink, on 12 to 18 inch stems. May. One of the finest native flowers................. for $75 \mathrm{c}$

YUCCA-(Yucca filamentosa). Drooping, sword-like foliage, with flower stem much branched and closely packed with creamy-white bloom, rising 4 to 5 feet

$50 \mathrm{c}$ each

ZEBRA GRASS-(Miscanthus). A hardy, ornamental grass, with transverse stripes of white on the light green leaves. 4 to 5 feet. Tall plumes are produced late in the fall, which are suitable for winter bouquets. 50 c clump; 3 for $\$ 1.25$.

\section{Lilies}

CANADENSE-(Wild Meadow Lily)-Dainty yellow or yellowish red flowers on two to four foot stems. For moist, rich but well drained soil........25c each

CANDIDUM-(Madonna)-Large white, fragrant. Blooms in June with many waxy flowers on the tall spike; turns yellow and dies down after blooming. In August or September the silvery leaves appear for a winter protection. Must not be watered at any stage of growth. Plant in a well-drained sunny situation. $40 \mathrm{c}$ each, $\$ 3.50 \mathrm{doz}$; second size, 25c each, $\$ 2.50 \mathrm{doz}$. 
REGALE LILY-(Lilium regale). The new lily from China. A clear lustrous white, with yellow tints in the throat and pinkish tint on the outside of the long petals. A larger, better flower than $\mathrm{L}$. candidum, with stalks of mature plants often reaching five feet and with 8 to 12 flowers on a stalk. Very hardy. Blooming size..50c Small 2 yr. bulbs .................

REGAL LILY SEED . ..............50c per 100 SPECIOSUM RUBRUM-(Japanese)-Blooms in August. White, flushed red with spots of deep chocolate. 35 c each; $\$ 3.50$ per doz.

SPECIOSUM ALBUM-White form of above; $35 \mathrm{c}$ each.

SPLENDENS-(Flame Lily)-Large crowns of 7 to 9 deep flame-colored lilies on medium spike. Planted along an evergreen hedge, the fine effect is attractive. $25 c$ each; $\$ 2.50$ per doz.

TIGRINUM-The old-fashioned Tiger Lily of our mother's garden, always reliable and blooming at a time when other flowers are scarce...20 each; $\$ 2.00$ per doz.

\section{ISMENE}

ISMENE-(Hymenocallis calathena). Peruvian Daffodil. White, large, lily-like flowers, with several of the outer petals very long and curved. Heavy fragrance. Grows readily in the garden, but must be lifted and wintered the same as Gladioli. Large bulbs. . 3 for $\$ 1.00$

\section{MONTBRETIA}

MONTBRETIA-Golden orange, dainty flowers for cutting. Treat like Gladioli.............. for $25 \mathrm{c}$

\section{TIGRIDIA PAVONIA}

TIGRIDIA PAVONIA-Shell flower. Light orange, shell shape flower with brown spots. Must be kept warmer during the winter than gladioli and planted out when the ground is well warmed in the spring... \$2.50 doz.

\section{CHRISTMAS CACTUS}

This old-fashioned house-plant always blooms for us at Christmas. We set the pots in the ground in a sunny place for the summer, take them inside and place in a south window, and they always show buds by Thanksgiving. As they are plants from the desert, they do not want much water but do like the hot sun. Small plants that should bloom next Christmas, 35c each. 


\section{Member}

American Peony Society.

American Iris Society.

American Association of Nurserymen.

Illinois State Nurserymen's Association.

American Peony and Iris Growers Association. 


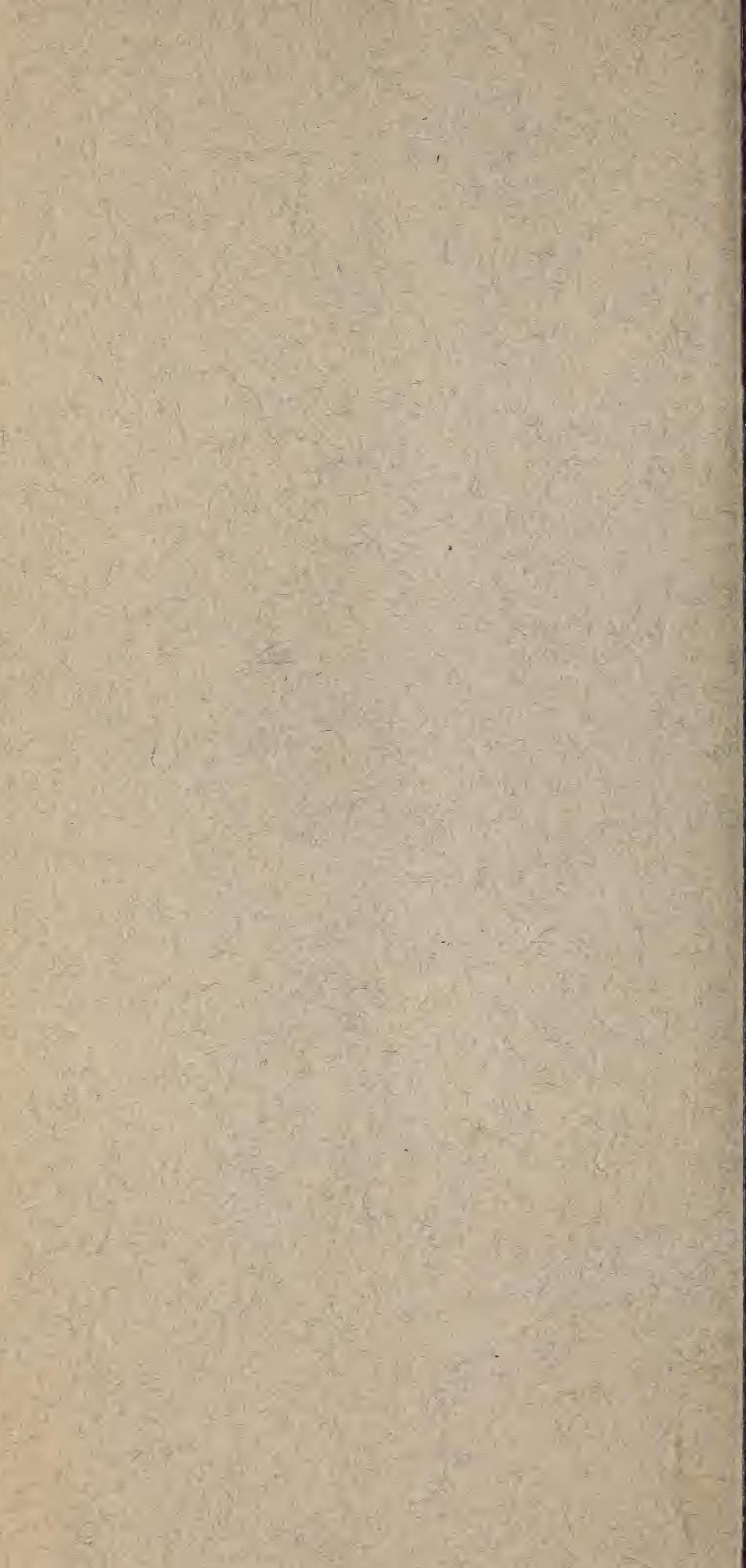

